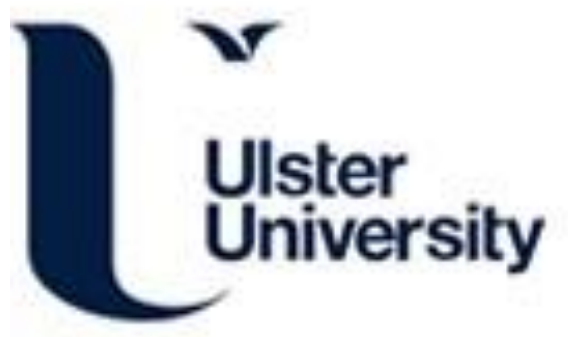

\title{
Structural determinants underlying the high efficacy of synaptic transmission and plasticity at synaptic boutons in layer 4 of the adult rat 'barrel cortex'
}

Rollenhagen, A., Klook, K., Saetzler, K., Qi, G., Anstötz, M., Feldmeyer, D., \& Lübke, J. H. R. (2015). Structural determinants underlying the high efficacy of synaptic transmission and plasticity at synaptic boutons in layer 4 of the adult rat 'barrel cortex'. Brain Structure and Function, 220(6), 3185-3209. https://doi.org/10.1007/s00429014-0850-5

Link to publication record in Ulster University Research Portal

Published in:

Brain Structure and Function

Publication Status:

Published (in print/issue): 01/11/2015

DOI:

$10.1007 / \mathrm{s} 00429-014-0850-5$

Document Version

Publisher's PDF, also known as Version of record

\section{General rights}

Copyright for the publications made accessible via Ulster University's Research Portal is retained by the author(s) and / or other copyright owners and it is a condition of accessing these publications that users recognise and abide by the legal requirements associated with these rights.

\section{Take down policy}

The Research Portal is Ulster University's institutional repository that provides access to Ulster's research outputs. Every effort has been made to ensure that content in the Research Portal does not infringe any person's rights, or applicable UK laws. If you discover content in the Research Portal that you believe breaches copyright or violates any law, please contact pure-support@ulster.ac.uk. 


\title{
Structural determinants underlying the high efficacy of synaptic transmission and plasticity at synaptic boutons in layer 4 of the adult rat 'barrel cortex'
}

\author{
Astrid Rollenhagen · Kerstin Klook • \\ Kurt Sätzler · Guanxiao Qi · Max Anstötz • \\ Dirk Feldmeyer · Joachim H. R. Lübke
}

Received: 20 May 2014 / Accepted: 10 July 2014

(c) Springer-Verlag Berlin Heidelberg 2014

\begin{abstract}
Excitatory layer 4 (L4) neurons in the 'barrel field' of the rat somatosensory cortex represent an important component in thalamocortical information processing. However, no detailed information exists concerning the quantitative geometry of synaptic boutons terminating on these neurons. Thus, L4 synaptic boutons were investigated using serial ultrathin sections and subsequent quantitative 3D reconstructions. In particular, parameters representing structural correlates of synaptic transmission and plasticity such as the number, size and distribution of pre- and postsynaptic densities forming the active zone (AZ) and of the three
\end{abstract}

A. Rollenhagen, K. Klook and J. H. R. Lübke have equally contributed to this work.

Electronic supplementary material The online version of this article (doi:10.1007/s00429-014-0850-5) contains supplementary material, which is available to authorized users.

A. Rollenhagen · K. Klook · G. Qi · M. Anstötz ·

D. Feldmeyer · J. H. R. Lübke ( $\square)$

Institute of Neuroscience and Medicine INM-2, Research Centre

Jülich GmbH, Leo-Brandt Str., 52425 Jülich, Germany

e-mail: j.luebke@fz-juelich.de

K. Klook

IRTG Graduate School 1328, RWTH/University Hospital,

Aachen, Germany

K. Sätzler

School of Biomedical Sciences, University of Ulster,

Cromore Rd., Londonderry BT52 1SA, UK

D. Feldmeyer · J. H. R. Lübke

Department of Psychiatry, Psychotherapy and Psychosomatics,

Medical Faculty, RWTH/University Hospital, Pauwelstr. 30,

52074 Aachen, Germany

D. Feldmeyer · J. H. R. Lübke

JARA Translational Brain Medicine, Jülich/Aachen, Germany functionally defined pools of synaptic vesicles were analyzed. L4 synaptic boutons varied substantially in shape and size; the majority had a single, but large AZ with opposing pre- and postsynaptic densities that matched perfectly in size and position. More than a third of the examined boutons showed perforations of the postsynaptic density. Synaptic boutons contained on average a total pool of $561 \pm 108$ vesicles, with $\sim 5 \%$ constituting the putative readily releasable, $\sim 23 \%$ the recycling, and the remainder the reserve pool. These pools are comparably larger than other characterized central synapses. Synaptic complexes were surrounded by a dense network of fine astrocytic processes that reached as far as the synaptic cleft, thus regulating the temporal and spatial glutamate concentration, and thereby shaping the unitary EPSP amplitude. In summary, the geometry and size of AZs, the comparably large readily releasable and recycling pools, together with the tight astrocytic ensheathment, may explain and contribute to the high release probability, efficacy and modulation of synaptic transmission at excitatory L4 synaptic boutons. Moreover, the structural variability as indicated by the geometry of L4 synaptic boutons, the presence of mitochondria and the size and shape of the AZs strongly suggest that synaptic reliability, strength and plasticity is governed and modulated individually at excitatory L4 synaptic boutons.

Keywords Barrel cortex - Layer 4 synaptic boutons . Quantitative 3D reconstructions - Distinct pools of synaptic vesicles $\cdot$ Synaptic transmission and plasticity

\section{Introduction}

In most sensory cortices, layer 4 (L4) is the main input station for thalamocortical afferents originating from the respective thalamic relay nuclei (for review see Sherman 
and Guillery 1996). The main recipients of these afferents in the 'barrel field' of the rat somatosensory cortex are excitatory L4 spiny neurons, namely spiny stellate and star pyramidal neurons (Simons and Woolsey 1984; Benshalom and White 1986; Lübke et al. 2000; Meyer et al. 2010; Schoonover et al. 2014) although a fraction also terminates on layer 5 and 6 pyramidal neurons (Constantinople and Bruno 2013; Ramirez et al. 2014), and on aspinous, presumably GABAergic interneurons (White et al. 1984; Benshalom and White 1986; Ahmed et al. 1994, 1997). Thus, excitatory L4 neurons have long been regarded as the first station of intracortical information processing (Harris and Woolsey 1983; Armstrong-James et al. 1992; Jacobs and Juliano 1995; Feldmeyer et al. 1999; Bruno and Sakmann 2006; but see Constantinople and Bruno 2013).

An understanding of the function of excitatory L4 spiny neurons in the microcircuitry of the 'barrel field' requires a detailed knowledge of their structural and functional properties and synaptic connectivity. It has been shown that intra- and translaminar layer 4 excitatory and inhibitory synaptic connections are very reliable, as indicated by relatively low failure rates, low coefficients of variation, but also by differences in short-term plasticity (Feldmeyer et al. 1999, 2002, 2005; Egger et al. 1999; Beierlein et al. 2003; Silver et al. 2003; Helmstaedter et al. 2008, 2009; Koelbl et al. 2013). In particular, excitatory connections between L4 spiny stellate neurons are characterized by their high synaptic efficacy, strength and reliability. High frequency bursts of presynaptic action potentials (APs) can evoke suprathreshold postsynaptic APs (Feldmeyer et al. 1999), a phenomenon never observed in other cortical connections.

In contrast to functional studies of layer 4 connections (Feldmeyer et al. 1999, 2005, 2006; Egger et al. 1999; Silver et al. 2003; Ramirez et al. 2014; for review see Feldmeyer et al. 2013), relatively little is known about the quantitative geometry of intracortical synaptic boutons terminating on L4 excitatory neurons. However, for a detailed, improved understanding of the signal cascades underlying synaptic transmission and plasticity in layer 4, a quantitative morphological analysis of the pre- and postsynaptic structures is of importance. In particular, realistic values of the number, geometry and distribution of actives zones (AZs; transmitter release sites) together with detailed information concerning the size and organization of the three functionally defined pools of synaptic vesicles, namely the readily releasable, recycling and reserve pools, provide important constraints for realistic models and numerical simulations of various parameters of synaptic transmission that are to date not available.

Thus, the quantitative geometry of intracortical synaptic boutons terminating on L4 excitatory neurons was examined to determine the structural factors underlying their unique functional properties. The analysis was based on large series of ultrathin sections and digital electron microscopic (EM) images through the dendritic domain of identified biocytin-filled spiny stellate neurons and on dendritic segments of excitatory L4 neurons in perfusionfixed and conventionally processed and embedded rat brains. Furthermore, to investigate the glial coverage of synaptic boutons in layer 4 , pre-embedding glutamine synthetase immunohistochemistry was carried out through individual 'barrels' of the rat somatosensory cortex. Subsequently, computer-assisted 3D reconstructions were performed for further quantification and correlation of various parameters that represent structural correlates of synaptic transmission and plasticity.

Here, we demonstrate for the first time in great detail, that several structural features of intracortical L4 synaptic boutons differ fundamentally from those estimated for other central synapses for which quantitative data are available, such as the calyx of Held (Rowland et al. 2000; Nicol and Walmsley 2002; Sätzler et al. 2002), the cerebellar (Xu-Friedman et al. 2001; Xu-Friedman and Regehr 2003) and hippocampal mossy fiber boutons (MFB) (Rollenhagen et al. 2007), the thalamic GABAergic synaptic boutons (Wanaverbecq et al. 2008), and other central synapses examined previously (reviewed by Rollenhagen and Lübke 2006).

The correlation of the detailed structural geometry and functional data of intracortical L4 synaptic boutons presented here as the first description of a quantitative model of a cortical synapse will help to understand various parameters of synaptic transmission and plasticity in the neocortex. Hence, this study will contribute and add to a much better understanding of the behavior of synapses embedded in different neuronal microcircuits in the normal and pathologically altered brain.

\section{Materials and methods}

All experimental procedures were approved by the Animal Research Committee of the Research Centre Jülich GmbH and complied with the guidelines laid out in the EU directive regarding the protection of animals used for experimental and scientific purposes.

\section{Electrophysiology}

\section{Brain slices and solutions}

Wistar rats (24-30 days old) were decapitated and slices through the somatosensory cortex were cut in cold extracellular solution using a vibrating microslicer (Mikrom HM $650 \mathrm{~V}$, Thermo Scientific, Germany). The method 
described by Agmon and Connors (1991) established for mouse barrel cortex was used with minor modifications required for the rat brain (Radnikow et al. 2012). The tissue was sliced at slow speed and high vibration frequency into $300 \mu \mathrm{m}$-thick 'semicoronal' slices (Land and Kandler 2002). They were then collected with a pipette and subsequently incubated $(30-60 \mathrm{~min})$ in an artificial saline solution bubbled with $95 \% \mathrm{O}_{2}$ and $5 \% \mathrm{CO}_{2}$ containing $6 \mathrm{mM} \mathrm{MgCl}_{2}$ to reduce synaptic activity before recording at room temperature $\left(22-24{ }^{\circ} \mathrm{C}\right)$. After pre-incubation, slices were transferred to the experimental setup and selected for best visibility of 'barrels' using infrared gradient contrast (IR-DIC) microcopy. During the experiment, slices were continuously superfused with an extracellular solution containing (in $\mathrm{mM}$ ): $125 \mathrm{NaCl}, 2.5 \mathrm{KCl}, 25$ glucose, $25 \mathrm{NaHCO}_{3}, 1.25 \mathrm{NaH}_{2} \mathrm{PO}_{4}, 2 \mathrm{CaCl}_{2}$, and $1 \mathrm{MgCl}_{2}$, and bubbled with $95 \% \mathrm{O}_{2}$ and $5 \% \mathrm{CO}_{2}$. The intracellular pipette solution used for both the pre- and postsynaptic neuron had the following composition (in $\mathrm{mM}$ ): 135 potassium gluconate, $4 \mathrm{KCl}, 10 \mathrm{HEPES}, 10$ phosphocreatine-Na, 4 ATP-Mg (pH 7.2, osmolarity 291 mOsm). Biocytin (Sigma, Munich, Germany) at a concentration of $1 \mathrm{mg} / \mathrm{ml}$ was routinely added to the internal solution. Slices were placed in the recording chamber under an upright microscope (Axioskop FS1, Carl Zeiss, Göttingen, Germany; fitted with w2.5 plan/0.075 NA and w40W/0.80 objectives) with the pial surface pointing to the front and the hippocampus to the right.

The 'barrel' field was visualized at low magnification under bright-field illumination and can be identified in layer 4 as narrow dark stripes with evenly spaced, light 'hollows' (Agmon and Connors 1991; Feldmeyer et al. 1999). 'Barrel' structures are present in six or seven slices, but a continuous band of 'barrels' is visible only in two or three slices just above the fimbria-fornix and the lateral ventricle. Individual excitatory L4 neurons were identified at $40 \times$ magnification using IR-DIC microscopy.

\section{Electrophysiological recordings}

Whole-cell voltage recordings from synaptically coupled pairs of neurons were made at $33-36{ }^{\circ} \mathrm{C}$ using patch pipettes of 4-8 M $\Omega$ resistance pulled of thick borosilicate glass capillaries (outer diameter $2.0 \mathrm{~mm}$; inner diameter $1.0 \mathrm{~mm}$ ). Biocytin was included in the recording solution for subsequent identification and morphological analysis of the recorded neurons. After patching the postsynaptic neuron, connections were searched for using extracellular 'loose-seal' stimulation of neighboring L4 neurons. When an action potential (AP) was evoked by loose-seal stimulation, this was visible as a small deflection on the voltage trace. When the AP resulted in an excitatory postsynaptic potential (EPSP) in the postsynaptic neuron at short latency (i.e. within 1-2 ms), the 'searching' pipette was withdrawn. The presynaptic cell was then re-patched with a new recording pipette $(5-8 \mathrm{M} \Omega)$ filled with biocytin-containing intracellular solution, and APs were elicited in the whole-cell (current-clamp) mode. Signals were amplified using an EPC10-triple patch clamp amplifier (HEKA Elektronik, Lambrecht, Germany), filtered at $2.9 \mathrm{kHz}$, and sampled at $10 \mathrm{kHz}$ (see also Feldmeyer et al. 1999). Acquired data were stored on the hard disk of a Macintosh computer for off-line analysis (WaveMetrics, Lake Oswego, Oregon, USA). For all data mean \pm SD were given.

\section{Synaptic pharmacology}

In some experiments aimed at determining the size of the $\alpha$-amino-3-hydroxy-5-methyl-4-isoxazolepropionic acid (AMPA) or $N$-methyl-D-aspartate (NMDA) component of the EPSP, $10 \mu \mathrm{M}$ 6-cyano-7-nitroquinoxaline-2,3-dione (CNQX) or $50 \mu \mathrm{M}(2 \mathrm{R}$ )-amino-5-phosphonovaleric acid (AP5) was added to the extracellular solution, respectively. For control conditions, 10-20 sweeps were recorded. After adding the glutamate receptor antagonists CNQX or AP5 to the extracellular solution, consecutive sweeps were recorded until the EPSP amplitude became stable. Last, the antagonist-containing ACSF was washed out and EPSPs were recorded until at least a partial washout of the drug was achieved.

\section{Synaptic physiology}

EPSP amplitude, latency and time course were determined as described previously (Feldmeyer et al. 1999) following a visual inspection of the recordings. The paired-pulse ratio (PPR) was defined as the 2nd mean EPSP amplitude divided by the 1st mean EPSP amplitude elicited by pairs of APs.

After recording, slices containing the biocytin-filled neurons were immersion-fixed with $4 \%$ paraformaldehyde and $0.1 \%$ glutaraldehyde diluted in $0.1 \mathrm{M}$ phosphate buffer (PB; pH 7.4) overnight and then further processed for electron microscopy as described in detail below for the perfusion-fixed material.

Structural investigation and analysis

Fixation and tissue processing for $3 D$ reconstructions of intracortical synaptic boutons in layer 4

For the reconstruction of individual intracortical L4 synaptic boutons and their postsynaptic target structures, three different experimental approaches were used: (1) immersion-fixed acute slices containing the recorded and 
a

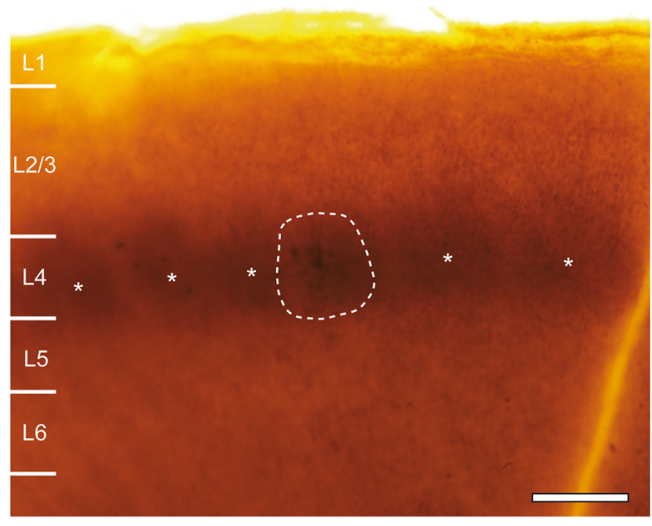

b

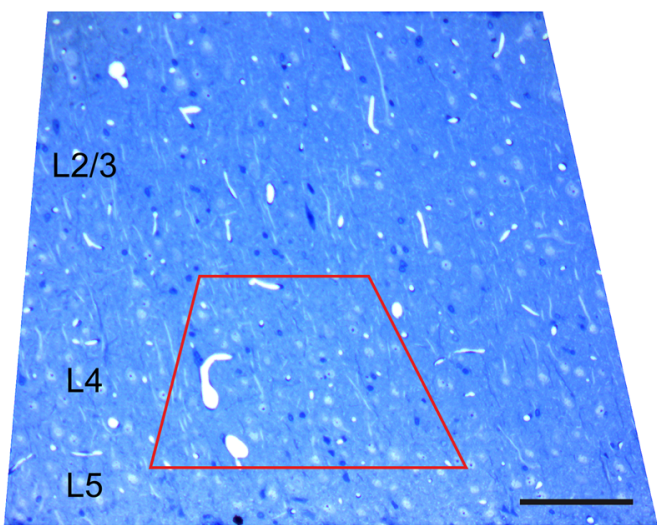

C

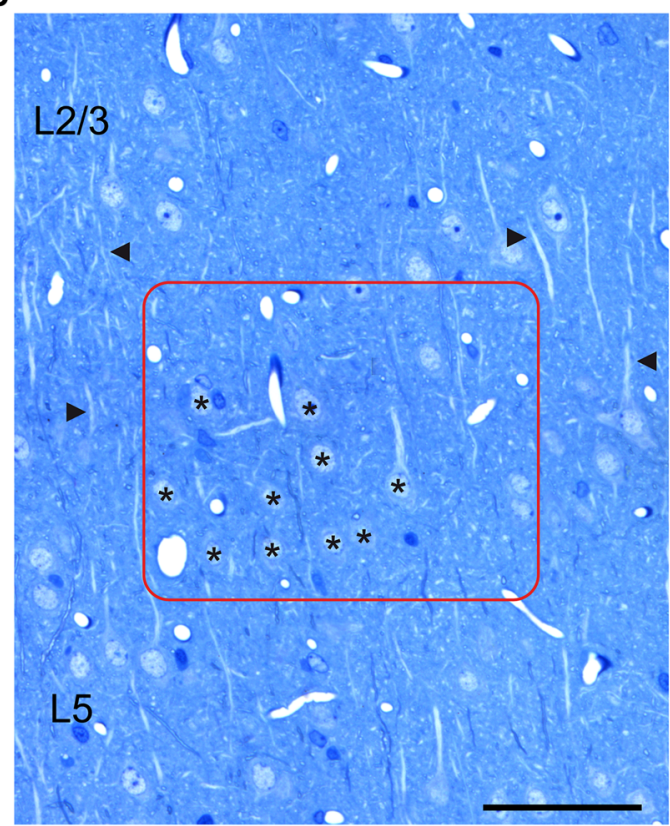

biocytin-filled excitatory L4 spiny neurons (Fig. 1a; $n=5$ ), which were then further processed according to the protocol described below. This experimental approach enables the reconstruction and further quantification of
4Fig. 1 Methodological approach to identify layer 4 in the EM processed material. a Low power light micrograph of an immersionfixed acute slice containing a biocytin-filled neuron located in an individual barrel (dashed outline) taken from a P30 day-old rat processed for EM. Note that additional barrels within layer 4 (marked by asterisks) are clearly visible as dark hollows in the osmium-treated slice. Scale bar $250 \mu \mathrm{m}$. b Toluidine blue stained semithin section (frontal plane) to identify layer 4 . The red trapezoid marks the area of the block at the level of layer 4 that was trimmed out for subsequent ultrathin sectioning. Scale bar $200 \mu \mathrm{m}$. c High power light micrograph of a toluidine blue stained semithin section at the level of layer 4. The red box outlines a single barrel. Note the typical clustered group of layer 4 excitatory neurons as indicated by asterisks and the bundled arrangement of the apical dendrites (marked by the four arrowheads) of layer 5 pyramidal neurons in the septa between barrels. Scale bar $200 \mu \mathrm{m}$

intracortical L4 synaptic boutons on electrophysiologically and morphologically identified synaptically coupled excitatory L4 spiny neurons (Figs. 1a, 2a1, a2, 3). One major disadvantage, however, is that the conversion of biocytin into an electron-dense 3'3 diaminobenzidine (DAB) reaction product obscures the entire postsynaptic density (Fig. 3b-e), one critical and important structural parameter for synaptic transmission and plasticity. Another critical factor is the time of the electrophysiological experiment and subsequent immersion fixation that could lead to alterations in the preservation of the ultrastructure. Thus, two additional experimental approaches were used: (2) perfusion-fixed brains of six adult Wistar rats embedded for conventional EM which guarantees an optimal ultrastructural preservation, and (3) perfusion-fixed brains of three adult Wistar rats where pre-embedding glutamine synthetase immunohistochemistry was carried out to investigate the astrocytic coverage of intracortical L4 synaptic boutons and their postsynaptic target structures.

To look for intra- and inter-individual differences not only in different animals, but also in different tissue blocks through individual 'barrels' mainly from the C-row of the 'barrel field' of the same animal were examined.

For the experiments described under (2) and (3), animals were deeply anesthetized with Narkodorm ${ }^{\circledR}(60 \mathrm{mg} / \mathrm{kg}$ body weight) and then transcardially perfused with physiological saline followed by an ice-cold 0.1 M PB (pH 7.4) fixative containing $4 \%$ paraformaldehyde and $0.1 \%$ glutaraldehyde (Polyscience Europe GmbH, Eppelheim, Germany) for 20-25 min. After $1 \mathrm{~h}$ of post-fixation, brains were removed and stored overnight in fresh fixative at $4{ }^{\circ} \mathrm{C}$. Serial 150 or $200 \mu \mathrm{m}$ thick vibratome sections (VT1000S Leica Microsystems, Nussloch, Germany) were cut in the frontal (coronal) plane through the 'barrel field' of the somatosensory neocortex. Sections were then postfixed for $1 \mathrm{~h}$ in 0.5 or $1 \%$ osmium tetroxide diluted in $0.1 \mathrm{M}$ PB-buffered sucrose ( $\mathrm{pH}$ 7.4) at room temperature for 30-60 $\mathrm{min}$ (in the dark). Thereafter, they were 
dehydrated in an ascending series of ethanol $(20,30,50$, $60,70,80,90,96 \%, 10 \mathrm{~min}$ for each step and two $30 \mathrm{~min}$ steps in absolute ethanol), followed by two brief incubations (1 min each) in propylene oxide. Sections were then transferred into a mixture of propylene oxide and Durcopan resin (2:1 for $1 \mathrm{~h}, 1: 1$ for $1 \mathrm{~h}$; Fluka, Neu-Ulm, Germany) and stored overnight in pure resin. The next day sections were flat-embedded in fresh Durcopan and polymerized at $60{ }^{\circ} \mathrm{C}$ for 2 days.

From the biocytin-filled and conventionally embedded material, blocks containing a single 'barrel' were trimmed out (Fig. 1a). Prior to serial ultrathin sectioning, semi-thin sections were cut, toluidine blue stained and examined light microscopically to identify the 'barrel field' (Fig. 1b). Care was taken to cut serial ultrathin sections through an individual cluster of excitatory neurons in a single barrel (Fig. 1c). Such a series was then further inspected at the EM level where biocytin-filled excitatory spiny neurons can easily be identified by the opaque $\mathrm{DAB}$ reaction product (Fig. 3), or by their characteristic appearance in conventionally processed material and tissue reacted for glutamine synthetase (Figs. 4a1-a4, 8; see also results).

\section{Glutamine synthetase pre-embedding immunohistochemistry}

To examine the astrocytic coverage at L4 synaptic boutons and their target structures, three adult Wistar rats were transcardially perfused as described above. After post-fixation, $100 \mu \mathrm{m}$ thick vibratome sections were cut in the frontal (coronal) plane. The 'barrel field' of the somatosensory cortex was dissected and then cryoprotected in $0.1 \mathrm{M}$ PB-buffered $10 \%$ (30 min), $20 \%$ (30 min) and $30 \%$ sucrose overnight. Sections were then freeze-thawed in liquid nitrogen, rinsed in $0.1 \mathrm{M} \mathrm{PB}$, blocked in $0.1 \mathrm{M}$ PB-buffered saline (PBS) containing $0.5 \%$ goat serum albumin $(1.5 \mathrm{~h})$ and finally incubated in a monoclonal mouse anti-glutamine synthetase antibody $(1: 1,000$; Chemicon Europe, Hampshire, UK) overnight at $4{ }^{\circ} \mathrm{C}$. Sections were thoroughly washed in $0.1 \mathrm{M}$ PBS, then incubated in biotinylated anti-mouse secondary antibody for $2 \mathrm{~h}$ (1:100, Vector, Linaris, Wertheim, Germany). After thorough washing in $0.1 \mathrm{M}$ PBS $(3 \times 10 \mathrm{~min}$ each $)$ sections were transferred into 0.1 M PBS-buffered ABC-elite solution for $2 \mathrm{~h}$ (1:100; Vector, Linaris, Wertheim, Germany). Thereafter, they were rinsed in $0.05 \mathrm{M}$ Tris-buffered saline (TBS) and reacted in 0.05 M TBS-buffered $\mathrm{DAB}$ for $10 \mathrm{~min}$. DAB reaction was stopped by $0.05 \mathrm{M}$ TBS, sections were transferred into $0.1 \mathrm{M}$ PBS, and then post-fixed in $0.1 \mathrm{M}$ sucrose PBS-buffered $0.5 \%$ osmium tetroxide (30 $\mathrm{min})$, dehydrated through an ascending series of ethanol, propylene oxide, and finally flat-embedded in Durcopan as described above.

\section{GABA post-embedding immunogold labeling}

The immunogold staining procedure was carried out as described by Somogyi and Hodgson (1985) using a commercially available antiserum against GABA (Sigma, München, Germany). The immunostaining was carried out on droplets of Millipore-filtered solutions in humid Petri dishes. Immersion in $1 \%$ periodate $(10 \mathrm{~min})$ was followed by several washes in double-distilled water. Subsequently, grids were transferred into a 2 or $5 \%$ sodium metaperiodate (10-30 min) solution, rinsed several times in doubledistilled water and followed by three times in $0.05 \mathrm{M}$ TBS (pH 7.4). Grids were then pre-incubated in $1 \%$ ovalbumin dissolved in $0.05 \mathrm{M}$ TBS (30 $\mathrm{min}$ ), and subsequently in rabbit anti-GABA antiserum (1:5,000, in $1 \%$ normal goat serum in TBS). After rinsing in $0.05 \mathrm{M}$ TBS and $50 \mathrm{mM}$ Tris buffer ( $\mathrm{pH} 7.4$ ) containing $1 \%$ bovine serum and $0.5 \%$ Tween $20(10 \mathrm{~min})$, grids were incubated in the secondary antibody (goat anti-rabbit IgG-coated colloidal gold, $10 \mathrm{~nm}$ ) for $2 \mathrm{~h}$ (diluted 1:10, in darkness). After rinsing in $2 \%$ glutaraldehyde $(10 \mathrm{~min})$, grids were washed again in double-distilled water and stained with $5 \%$ uranyl acetate $(10 \mathrm{~min})$ and lead citrate (Reynolds 1963) for 2 min. In control experiments without the primary antibody, sections processed for GABA postimmunogold labeling show almost no, or only very low, background labeling, whereas labeling of GABAergic structures clearly exceeded the mean gold particle density of the maximum background staining (by at least 4 standard deviations).

\section{Serial sectioning}

Serial ultrathin sections $(55 \pm 5 \mathrm{~nm}$ in thickness, silver to light gray interference contrast appearance) were cut on a LeicaUltracutS ultramicrotome (Leica Microsystems, Vienna, Austria) and collected on Formvar-coated slot copper grids. Individual series comprised 70-150 ultrathin sections to allow the reconstruction of synaptic boutons terminating on individual dendritic segments. Prior to EM examination, sections were stained with $5 \%$ aqueous uranyl acetate for $20 \mathrm{~min}$ and lead citrate for $5 \mathrm{~min}$ (Reynolds 1963).

\section{$3 D$ reconstructions}

Dendrites of excitatory L4 neurons within a single 'barrel' (Fig. 1c) were photographed with a Zeiss Libra 120 (Fa. Zeiss, Oberkochen, Germany) equipped with a Proscan $2 \mathrm{~K}$ digital camera and the SIS Analysis software (Olympus Soft Imaging System, Münster, Germany) at a primary magnification of $8,000 \times$. Digital images were then imported into the reconstruction software OpenCAR, stacked and transformed linearly such that corresponding 
structures were aligned along all consecutive images comprising the 3D image stack. In each image series, all structures of interest were defined, outlined and subsequent 3D-volumetric reconstructions were performed from which surface and volume measurements were obtained.

Within a given stack only 'complete' synaptic boutons were included in the sample and were selected using the following criteria: Endterminal boutons in most of the cases, part of the axons could be followed throughout the series of digital images leading into the opening of a synaptic bouton. The termination of a synaptic bouton was initiated by the decrease in size until the structure became too small to be visible in the following consecutive digital images. En passant boutons: here, the axons and boutons could be followed in both directions within a series (70-150) of ultrathin sections.

The surface areas of the pre- and postsynaptic densities were measured separately. The presynaptic density was computed by extracting those areas from the reconstructed presynaptic membranes that were covered by this membrane specialization. The size of the postsynaptic density opposing the presynaptic density at each synaptic contact (active zone) was estimated using the perimeter ratio between the outlines of the postsynaptic density to that of the synaptic contact.

The synaptic cleft width was measured at the two lateral edges and the center of the pre- and postsynaptic densities on digital electron microscopic images using the SIS software. Only synaptic boutons in which the AZ was cut in a perpendicular plane and which showed the typical broadening of the synaptic cleft were included in the sample ( $n=5$ animals, $n=150$ AZs). The two values of the estimated cleft width for the two lateral edges were averaged, and a mean \pm SD was calculated for each animal. Finally, a total mean $\pm \mathrm{SD}$ over all animals is given.

All calculations were performed off-line using a batch version of OpenCAR, which generates 3D reconstructions as well as space-delimited tables for each measurement that are readable by standard analysis software. For further details on 3D reconstructions see Sätzler et al. (2002).

Tissue shrinkage may affect the reported morphological parameters. However, it has recently been shown by high pressure freezing, cryo-substitution and subsequent EM, that no significant differences in quantitative parameters of synaptic structures as measured here were found when compared with conventionally embedded electron microscopic material (Zhao et al. 2012a, b). Thus, no correction for shrinkage was performed.

\section{Analyzing vesicle distribution and pool sizes}

To obtain estimates for the size of synaptic vesicles and distance distributions, each vesicle in a synaptic bouton was marked and its diameter and the minimal distance between its center of gravity and the presynaptic density of an individual AZ were determined. This distance diminished by one vesicle radius gives an estimate of the minimal 2D distance that a vesicle had to bridge before it 'touches' the membrane specialization. The minimal 2D distance for each vesicle to a presynaptic density was calculated. Thus, it was possible to define different pools of synaptic vesicles for each presynaptic density depending on the vesicle perimeter and by plotting their distribution.

Several potential errors in vesicle counting were considered. First, vesicle counting might be subjective in ultrathin sections with 'suboptimal' quality that could occur in such large series. To address this issue, digital images of relatively low quality were selected and vesicles were traced by different experimenters involved in this project (4 experimenters, 10 consecutive sections). Then final counts, diameter, and distance estimates were compared and the mean and SD was calculated, which gave an upper bound for this source of variability. The variability for the mean diameters and distances reported was $\pm 2.5 \mathrm{~nm}$ [coefficient of variation (CV), 0.16] and $\pm 4.2 \mathrm{~nm}$ $(\mathrm{CV}, 0.01)$, respectively. Second, errors in estimates of vesicle numbers may occur by double counts of individual vesicles in adjacent sections (Abercrombie 1946). To address the possibility of double counts of small clear vesicles, we simulated the appearance of these vesicles in ultrathin sections under conditions when the vesicle diameter was comparable with the thickness of the sections ( $\sim 50 \mathrm{~nm})$. These simulations revealed that synaptic vesicles showed a clear ring-like appearance in electron micrographs only if $\geq 50 \%$ of their volume was present in an ultrathin section (see also Sätzler et al. 2002). Because only clear ring-like structures were counted as vesicles, double counts were highly unlikely under these circumstances. Additionally, it is possible that vesicles are missed in densely packed regions, because ring-like traces partly overlap. This effect may counteract any double counts. Based on the small extent and the partially counteracting nature of the effects, the numbers of small clear vesicles reported in this study remained uncorrected (see also Rollenhagen et al. 2007). For large dense-core vesicles, double counts were excluded by careful examination of adjacent images and were only counted in the image where they appeared largest.

\section{Statistical analysis}

From the numerous 3D reconstructions and spreadsheets computed by OpenCAR, statistical summaries and graphs were generated automatically using special purpose functions written for the freely available statistics package $\mathrm{R}(\mathrm{R}$ Development Core Team. R: A language and environment 
for statistical computing. $\mathrm{R}$ Foundation for Statistical Computing, Vienna, Austria. ISBN 3-900051-07-0, URL http://www.R-project.org, 2005).

To assess differences in the distributions for the size of the pre- and postsynaptic densities, the non-parametric Kolmogorov-Smirnov test was used. In addition, the nonparametric Kruskal-Wallis $H$ test with Dunn's post hoc analysis was used to look for differences between experimental animals for the structural parameters analyzed. Results were considered significant if $p<0.05$. Where appropriate, $p$ values were explicitly specified. For all values, a median with the 1 st and 3rd quartile and a mean \pm SD over all animals are given (see Tables 1,2 ). For all correlations, the correlation coefficient $\left(R^{2}\right)$ and the Spearman correlation coefficient $\left(r_{\mathrm{s}}\right)$ are given.

\section{Results}

Reliable synaptic transmission between pairs of excitatory L4 spiny neurons

Dual whole-cell recordings were made from pairs of synaptically coupled L4 excitatory neurons ( $n=12$ pairs) in the 'barrel cortex' of postnatal 24 to 30 day old rats. During recording, the pre- and postsynaptic neurons were filled with biocytin to reveal their morphology (Fig. 2a1, a2). Subsequently neurons were processed for EM to analyze synaptic boutons terminating on functionally and morphologically identified excitatory L4 spiny neurons (Fig. 3; see also Tables 1, 2).

The majority of synaptically coupled excitatory neurons were spiny stellate cells with the characteristic asymmetrical dendritic arborization confined to a single 'barrel' (Fig. 2a1). The main axons were always seen to descend towards the white matter giving rise to numerous axonal collaterals, largely confined to the 'home column' in which the neurons were located. The majority of these collaterals formed a dense axonal plexus located in layer 4 itself and a second axonal domain within layer $2 / 3$, where they terminate or further ascend to layer 1 (Fig. 2a1). These collaterals were densely covered with synaptic boutons suggesting a high probability of innervation of the two main target regions (Feldmeyer et al. 1999; Lübke et al. 2000). Synaptic contacts (3-5 per connection) were found exclusively on dendrites, in particular at secondary and tertiary branches (Fig. 2a2) relatively close to the soma (see also Feldmeyer et al. 1999).

The pre- and postsynaptic neurons of a coupled pair were classified as excitatory on the basis of their AP discharge pattern (Fig. 2b1, b2). When stimulated with $1 \mathrm{~s}$ long depolarization steps, they responded initially with a high frequency of APs adapting to lower, but sustained frequencies. Thus, these neurons could be characterized as 'regular spiking' neurons (Feldmeyer et al. 1999; Staiger et al. 2004; this paper). Single presynaptic APs (Fig. 2c1) evoked unitary EPSPs (Fig. 2c2) with a peak amplitude of $1.02 \pm 1.33 \mathrm{mV} \quad$ (mean $\pm \mathrm{SD}$ ), a latency of $1.17 \pm 0.41 \mathrm{~ms}$, a rise time of $1.59 \pm 0.49 \mathrm{~ms}(20-80 \%)$, and a decay time constant of $36.4 \pm 15.4 \mathrm{~ms}$. At $32-33{ }^{\circ} \mathrm{C}$, the $\mathrm{CV}$ of the unitary EPSP amplitude was $0.37 \pm 0.16$ and the percentage of failures to evoke an unitary EPSP was $5.3 \pm 7.8 \%$. Both values decreased with increasing amplitude of the unitary EPSP (this paper; see also Feldmeyer et al. 1999).

Postsynaptic glutamate receptors in excitatory L4 spiny neurons were of the AMPA- and NMDA-type (Fig. 2d). At $-60 \mathrm{mV}$ in the presence of $1 \mathrm{mM} \mathrm{Mg}^{2+}$, NMDA receptors contributed with $27.0 \pm 9.9 \%$ to the unitary EPSP amplitude. In an $\mathrm{Mg}^{2+}$-free solution, the fractional NMDA receptor component of the EPSC was $46.2 \pm 34.4 \%$. All pairs investigated showed a weak frequency-dependent paired-pulse depression with a PPR of $0.92 \pm 0.14$ at $10 \mathrm{~Hz}$ (Fig. 2e).

These results suggest that synaptic transmission between pairs of excitatory L4 spiny neurons in young adult rats is very reliable, probably due to a high release probability and high synaptic efficacy, although a huge variability in shortterm plasticity was observed as indicated by their pairedpulse behavior.

We next investigated whether the unique functional properties of intralaminar excitatory L4 connections are reflected and explained by the structural composition of intracortical synaptic boutons terminating on excitatory L4 neurons.

Synaptic input in layer 4 of the 'barrel cortex'

Layer 4 is regarded as the main input station for signals from the sensory periphery (whisker pads) and receives both intrinsic intracortical and thalamocortical afferents projecting and establishing synaptic contacts with neurons in individual 'barrels' in layer 4 (but see Constantinople and Bruno 2013). The majority $(\sim 80 \%)$ of the intracortical axons originate from L4 excitatory spiny stellate and star pyramidal neurons as shown by paired- and multiple simultaneous whole-cell recordings and axon distribution analysis (Feldmeyer et al. 1999; Lübke et al. 2000, 2003). The other source of excitatory input arises from layer $6 \mathrm{a}$ pyramidal neurons (Zhang and Deschênes 1997; Kumar and Ohana 2008; Lee and Sherman 2008; for review see Lübke and Feldmeyer 2007) contributing to $\sim 8 \%$ to the total input. The remaining inputs are local axons of GABAergic interneurons in layer 4 (Staiger et al. 2009; Kätzel et al. 2011; Koelbl et al. 2013) or adjacent layer 2/3 (Helmstaedter et al. 2009; Meyer et al. 2011) and 

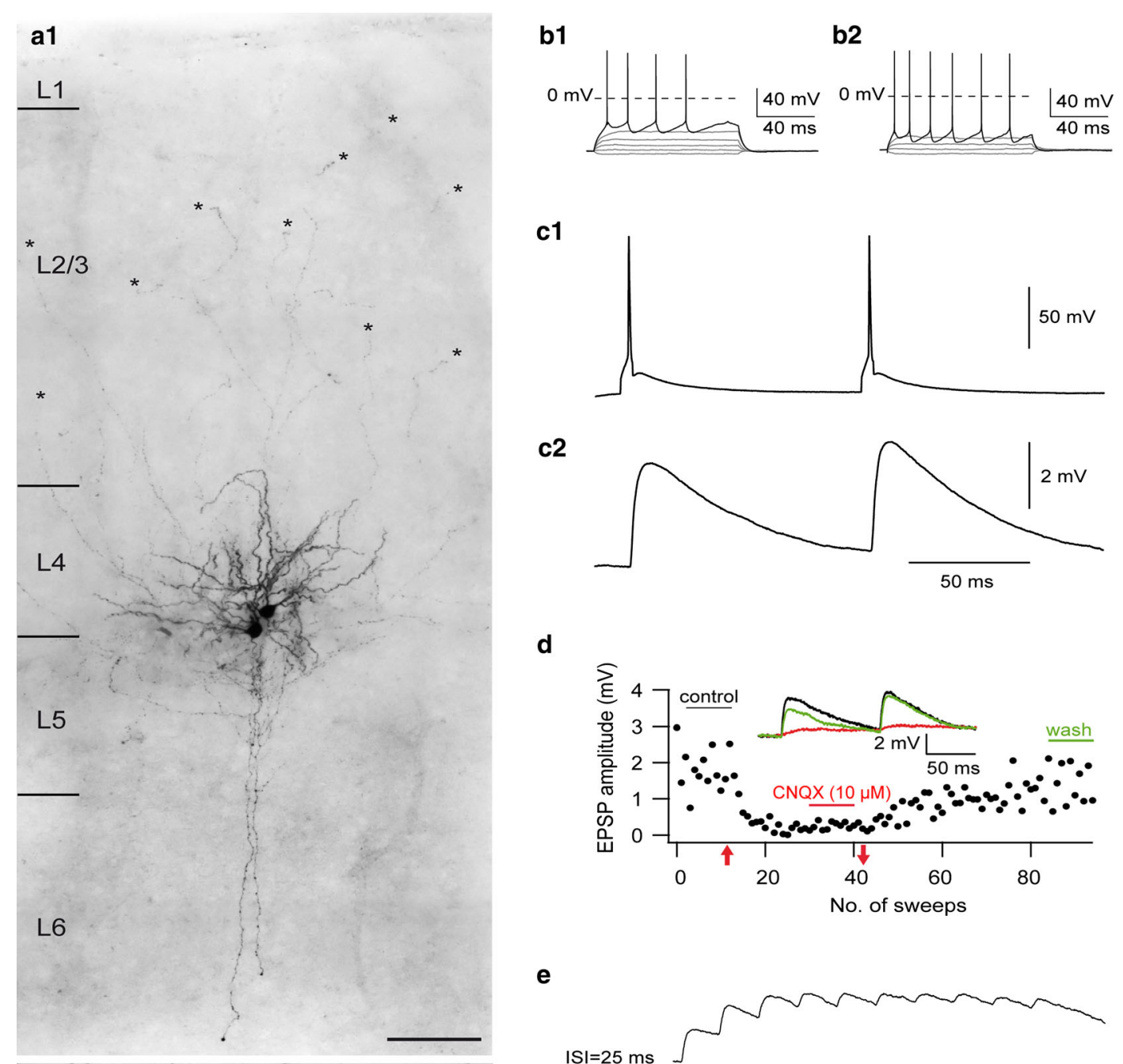

c1

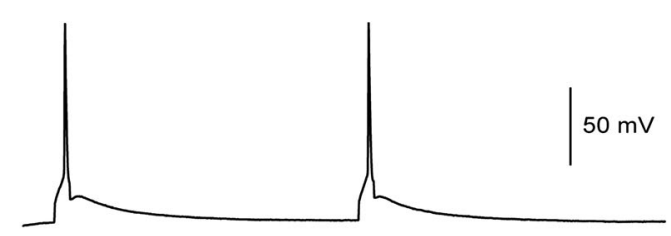

c2

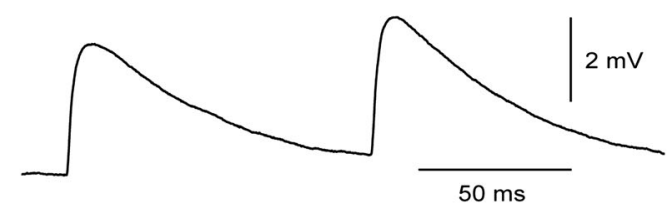

d

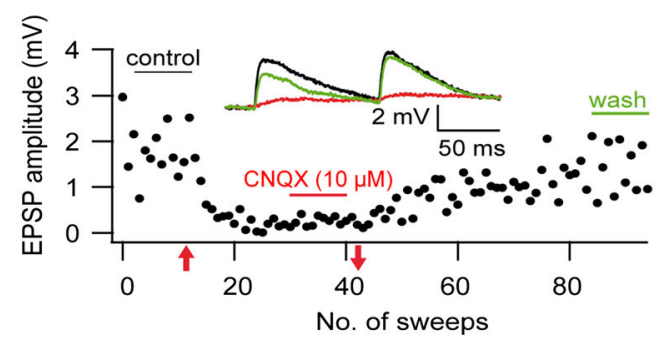

e
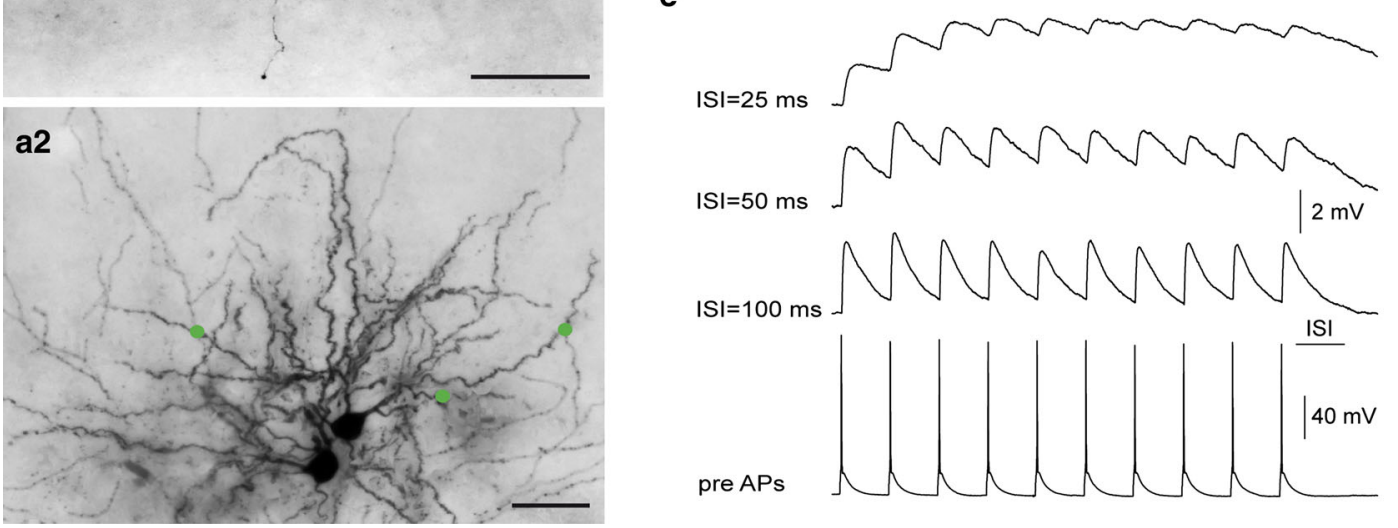

Fig. 2 Synaptic transmission and plasticity in excitatory L4-L4 connections. a1 Low power light microscopic image of a synaptically coupled pair of spiny stellate neurons filled with biocytin during recording. Note that the dendrites of the pre- and postsynaptic neurons are confined to layer 4 , whereas the majority of axonal collaterals (marked by black asterisks) were seen to ascend towards L1. The main axon projects deep into L6. Scale bar $100 \mu \mathrm{m}$. a2 High power light microscopic image of the dendritic configuration of the pre- (left) and postsynaptic (right) neuron. The axon of the presynaptic neuron established three putative, light microscopically identified synaptic contacts (green dots) on different dendritic locations of the postsynaptic neuron. Scale bar $50 \mu \mathrm{m}$. b1, b2 Regular action potential firing pattern of the pre- (b1) and postsynaptic (b2) neuron of the synaptically coupled pair shown in a1 and a2. c1, c2 Eliciting APs in the presynaptic

neuron (c1) resulted in evoked unitary EPSPs of different duration and amplitude in the postsynaptic neuron (c2). d Pharmacological properties of the pair shown in a1 and a2. Time course of the EPSP amplitude during pharmacological treatment. Inset mean EPSP waveforms from control (black trace), with $10 \mu \mathrm{M} \mathrm{CNQX} \mathrm{(red} \mathrm{trace)} \mathrm{and}$ after washout (green trace) are shown superimposed aligned with respect to the peak time of presynaptic APs. Each sweep was collected at $20 \mathrm{~s}$ intervals. e Frequency-dependent paired-pulse depression in the cell pair shown in $\mathbf{a} 1$ and $\mathbf{a} 2$. Note that the decrease in the interspike interval (ISI) resulted in a more pronounced paired-pulse depression (upper trace) in the postsynaptic neuron after evoking AP trains (bottom trace) in the presynaptic neuron (compare upper with the two middle traces) 
Fig. 3 Electron microscopy through the dendritic domain of a biocytin-filled L4 spiny stellate neuron. a Low power electron micrograph of a biocytin-labeled dendritic segment (dark DAB reaction product) at the branch point of a secondary dendrite into two tertiary branches. The synapses terminating on the dendritic shaft or spines were outlined as yellow contours. Scale bar $1 \mu \mathrm{m}$. b-d Three examples of a third order $(\mathbf{b}, \mathbf{c})$ and fourth order (d) dendritic segment of a biocytin-filled spiny stellate neuron with synapses established on both dendritic shafts and spines (yellow contours). e Example for a GABAergic synapse terminating on the dendritic shaft as revealed by the occurrence of gold grains distributed over the terminal. Scale bar in b-e, $0.5 \mu \mathrm{m}$
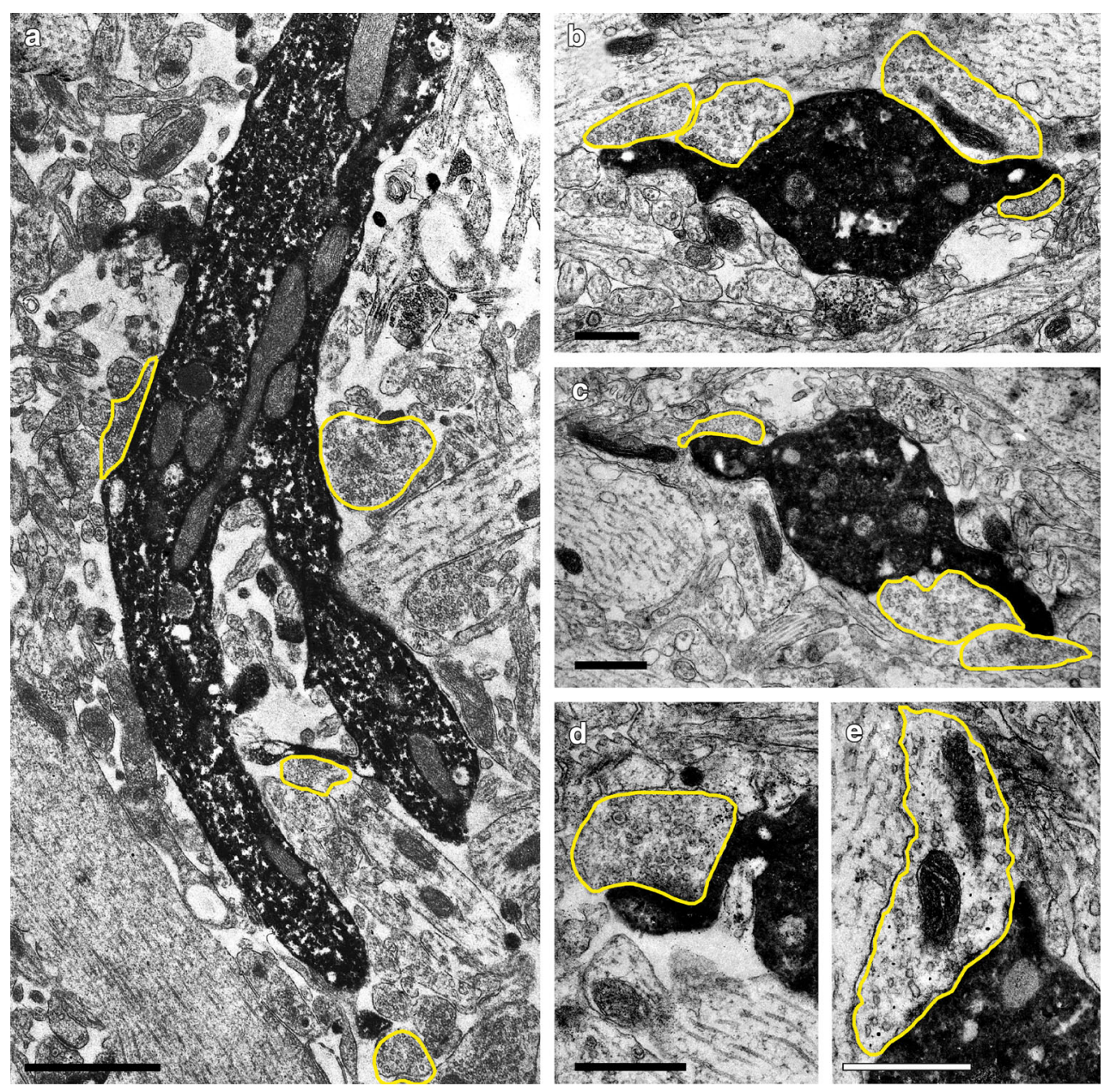

presumably layer 5 (Kätzel et al. 2011). The thalamic input originates from neurons of the ventroposterior medial nucleus (Benshalom and White 1986; Yu et al. 2006; Meyer et al. 2010), and terminates preferentially on somata and proximal portions of dendrites of L4 excitatory neurons. It has been demonstrated that these inputs contribute with $\sim 8 \%$ to the total synaptic input onto L4 excitatory neurons and also onto GABAergic interneurons (Benshalom and White 1986; Ahmed et al. 1994, 1997). Hence the majority of synaptic inputs are established by local axonal collaterals of excitatory L4 neurons.

Structural composition of intracortical L4 synaptic boutons and their postsynaptic targets

Serial ultrathin sections were always taken from a single 'barrel' (Fig. 1a) and through the dendritic domains of excitatory spiny L4 neurons (Figs. 1c, 2a2). In the case of the biocytin-filled material, dendritic segments were chosen where the majority of synaptic contacts were light microscopically identified in pairs of synaptically coupled neurons, namely second and third order dendrites
(Feldmeyer et al. 1999, this study). In the perfusion-fixed material, only dendritic segments showing the typical features of spines, characteristic for excitatory spiny neurons originally described using EM by Cragg $(1972,1975)$ and Peters and Kaiserman-Abramof (1969), were included in the sample (Figs. 3, 4). Finally, to avoid the possible overlap between thalamocortical and intracortical synaptic inputs, only dendritic segments located $\sim 50 \mu \mathrm{m}$ away from the somata were chosen for further analysis.

Taking the above defined criteria into account, a total of 252 synaptic boutons were completely reconstructed and analyzed (see Tables 1, 2). Thirty individual dendritic segments from different animals and blocks identified by the presence of typical spines (stubby, thin, mushroom and filipodial-like) characteristic for excitatory neurons were analyzed and completely reconstructed in serial digital EM images through the dendritic domain of excitatory L4 spiny neurons (Figs. 3, 4). No major significant differences for the structural parameters investigated here were found between biocytin-labeled (Fig. 3), conventionally embedded (Fig. 4a1-a4) and glutamine synthetase (Fig. 8) processed material as shown by statistical analysis comparing 

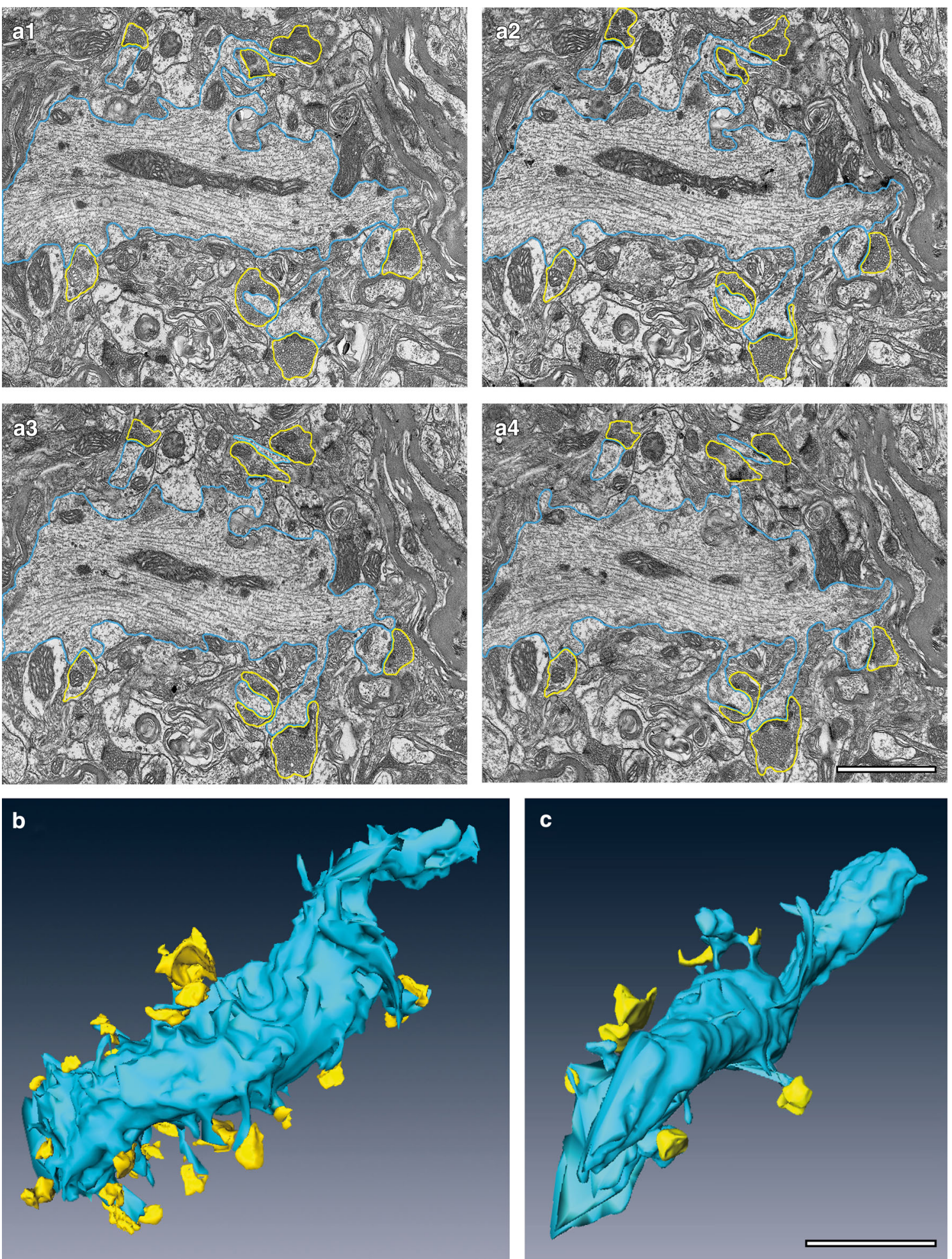

Fig. 4 Serial electron micrographs and 3D-volume reconstructions of intracortical L4 synaptic boutons. a1-a4 Four consecutive digital electron micrographs of a dendritic segment (outlined in light blue) of a L4 spiny stellate neuron with synaptic contacts (yellow contours) terminating on the dendritic shaft and spines. Scale bar $2 \mu \mathrm{m}$. b, c 3D-volume reconstructions of two individual dendritic segments (light blue) with terminating boutons (yellow). Note the differences in the number and size of boutons (some of which are hidden) along the dendritic segments (b 28 synapses on an $18 \mu \mathrm{m}$ long dendritic segment; c 9 boutons on a $14 \mu \mathrm{m}$ long dendritic segment). Scale bar $5 \mu \mathrm{m}$ 


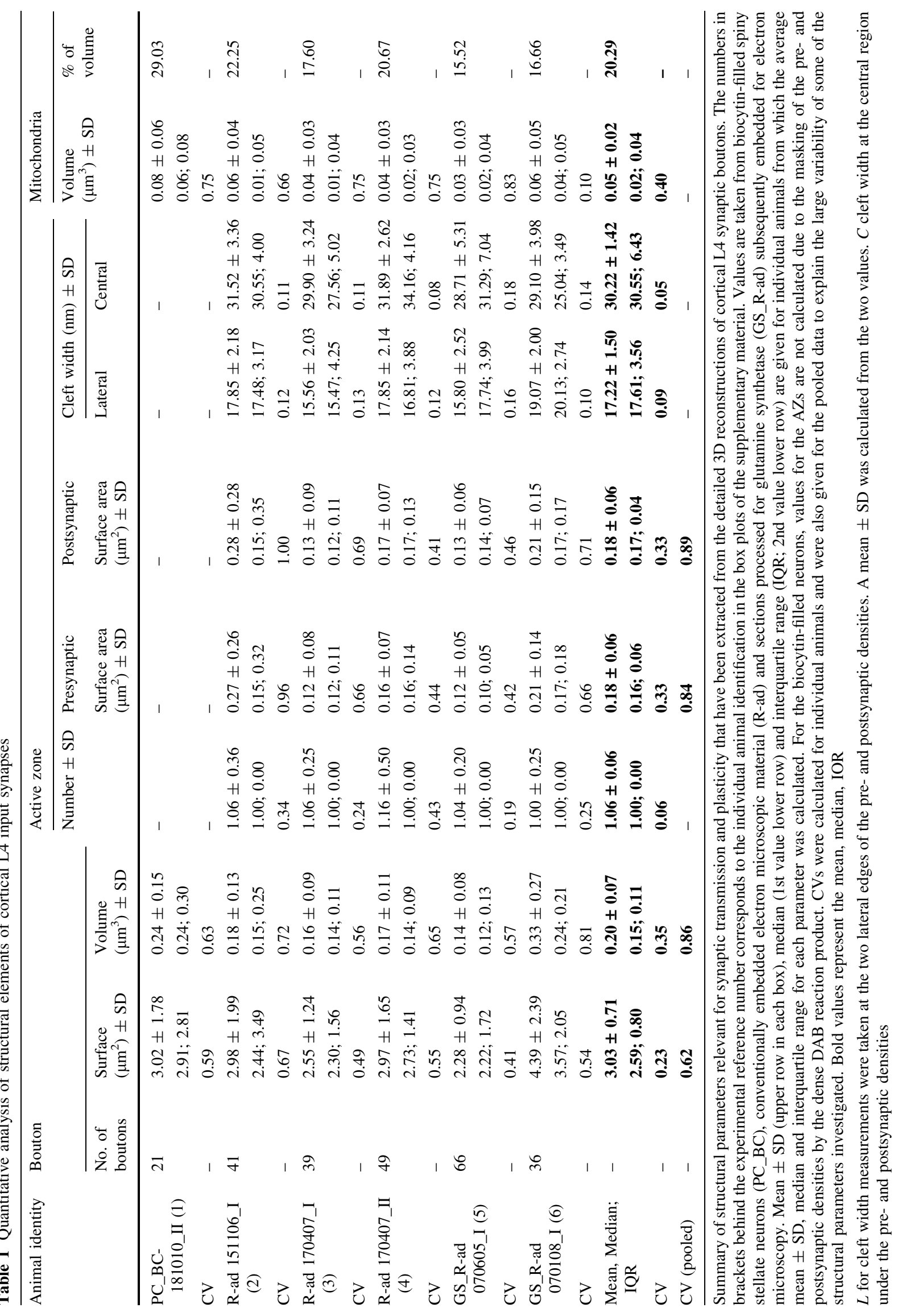




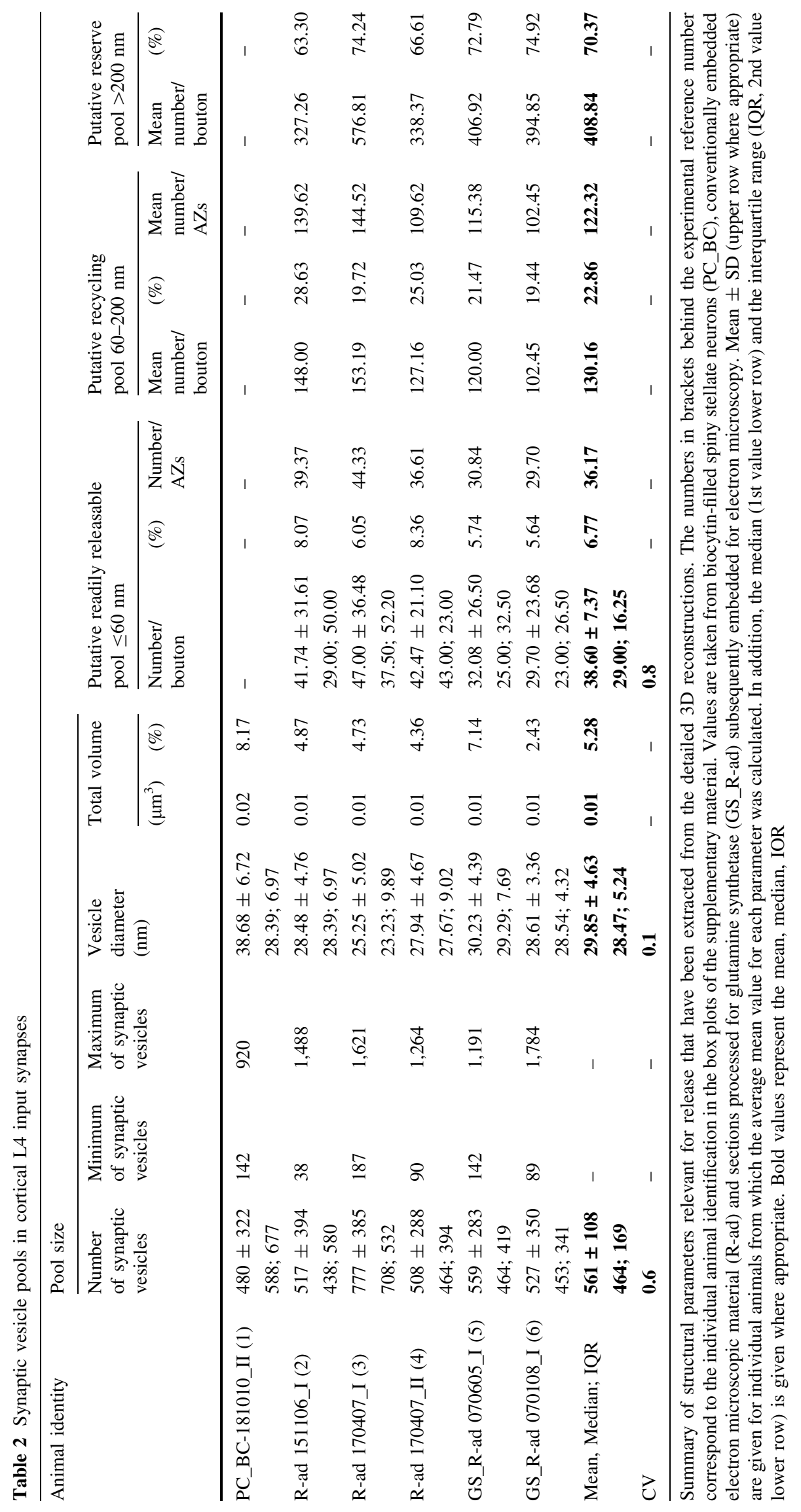



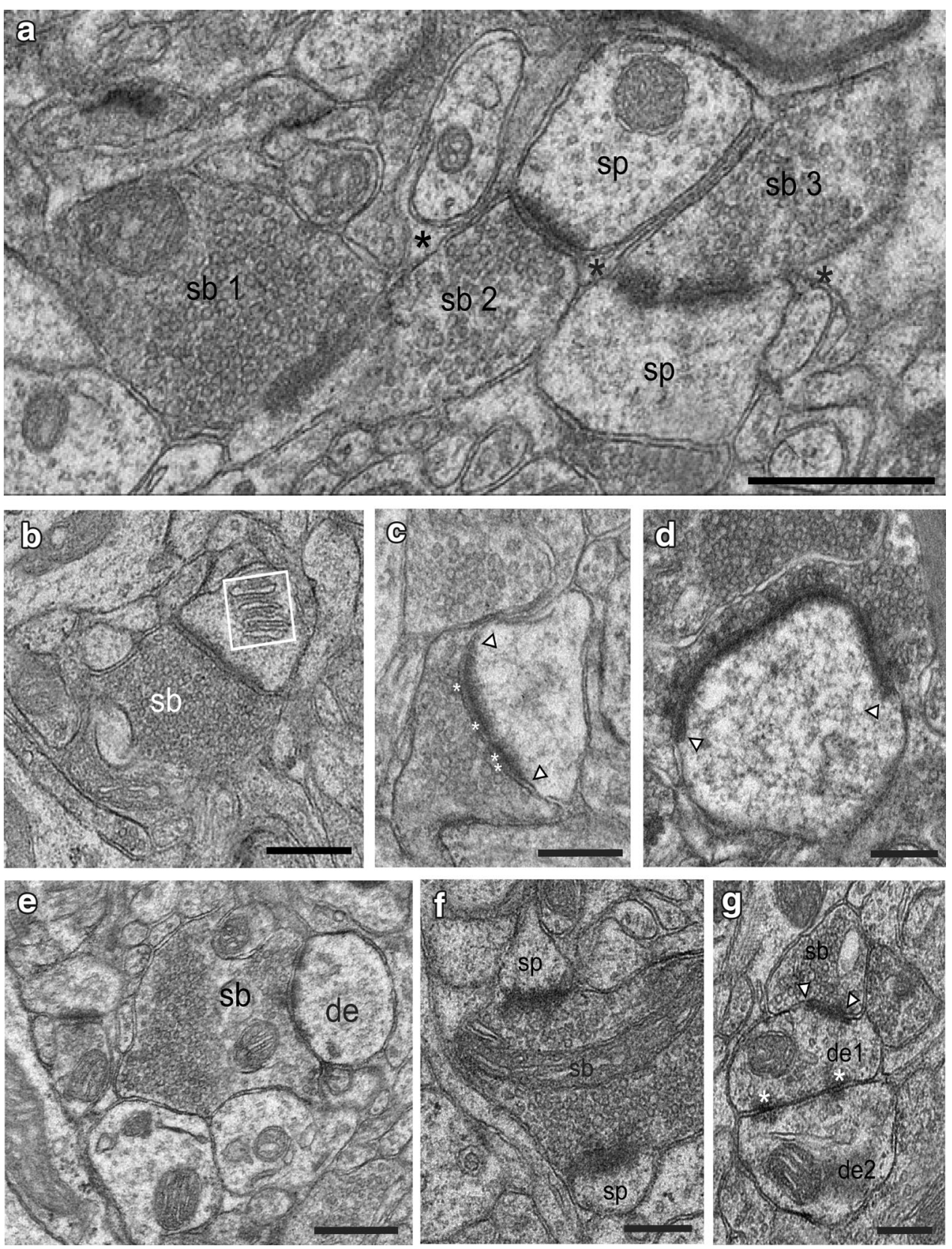

Fig. 5 Structural features of intracortical L4 synaptic boutons. a High power electron micrograph of three synaptic boutons (sb1sb3) establishing synaptic contacts with two dendritic spines ( $s p$ ). Note the difference in the size of the pool of synaptic vesicles between the three boutons. Black asterisks indicate adjacent astrocytic processes. Scale bar $1 \mu \mathrm{m}$. b Dendritic spine containing a large spine apparatus (frame area) with a synaptic bouton terminating on the spine head. Scale bar $0.5 \mu \mathrm{m}$. c Dendritic spine with macular, nonperforated pre- and postsynaptic density (marked by arrowheads) with a small endterminal synaptic bouton contacting the spine head. Multiple 'docked' vesicles are marked by asterisks. Scale bar $0.5 \mu \mathrm{m}$.

the experimental animals (see Suppl. Figs. 1-3, see also Materials and methods).

Axons established either en passant or endterminal synaptic boutons predominantly on dendritic spines d Synaptic bouton with a large pre- and postsynaptic density (marked by arrowheads) terminating on a dendritic spine. Note that the presynaptic density is perforated, whereas the postsynaptic density is non-perforated. Scale bar $0.5 \mu \mathrm{m}$. e Large synaptic bouton $(s b)$ terminating on a small caliber dendrite $(d e)$ with three distinct but adjacent AZs. Scale bar $1 \mu \mathrm{m}$. f Large synaptic bouton $(s b)$ terminating on two opposite dendritic spines $(s p)$. Scale bar $1 \mu \mathrm{m}$. g Dendro-dendritic synapse (marked by asterisks) between two small caliber dendrites $(d e)$. Interestingly, on the upper dendrite a synaptic contact is established by a small synaptic bouton $(s b)$. Scale bar $1 \mu \mathrm{m}$

(Figs. 3a-d, 4, 5a-d, f, 6) and, to a smaller fraction $(\sim 10 \%)$ on dendritic shafts of variable caliber (Figs. 3a, $5 \mathrm{e}, \mathrm{g})$. We found multiple kinds of spines belonging to the stubby (Figs. 4a1, 6a, g), mushroom (Figs. 4a2, a3, 6b-d, 
Fig. $63 \mathrm{D}$-volume

reconstructions of dendritic spines with AZs. a-

h Representative examples of dendritic spines found at L4 dendritic segments (light blue) of the stubby (a, g), mushroom $(\mathbf{b}, \mathbf{c}, \mathbf{d}, \mathbf{e})$, thin $(\mathbf{e})$ and filopodial-like (f) type. Note the differences in the shape and size of AZs (red areas) with a nonperforated $(\mathbf{a}-\mathbf{f})$ or perforated, ring-like appearance $(\mathbf{g}, \mathbf{h})$. Scale bar in $\mathbf{a}-\mathbf{h} 0.25 \mu \mathrm{m}$

Fig. 7 Distribution of the surface area of AZs. a Bar histogram showing the distribution of surface areas for all pre- (black columns) and postsynaptic densities (gray columns) analyzed.

b Corresponding cumulative histogram. Bin width, $0.01 \mu \mathrm{m}^{2}$. c Dot plot showing the correlation between the surface area of the pre- vs. the postsynaptic density. Note the nearly perfect correlation as indicated by the high $R^{2}$. d Dot plot showing the differences in surface area between the preand postsynaptic densities. The dashed line indicates the mean
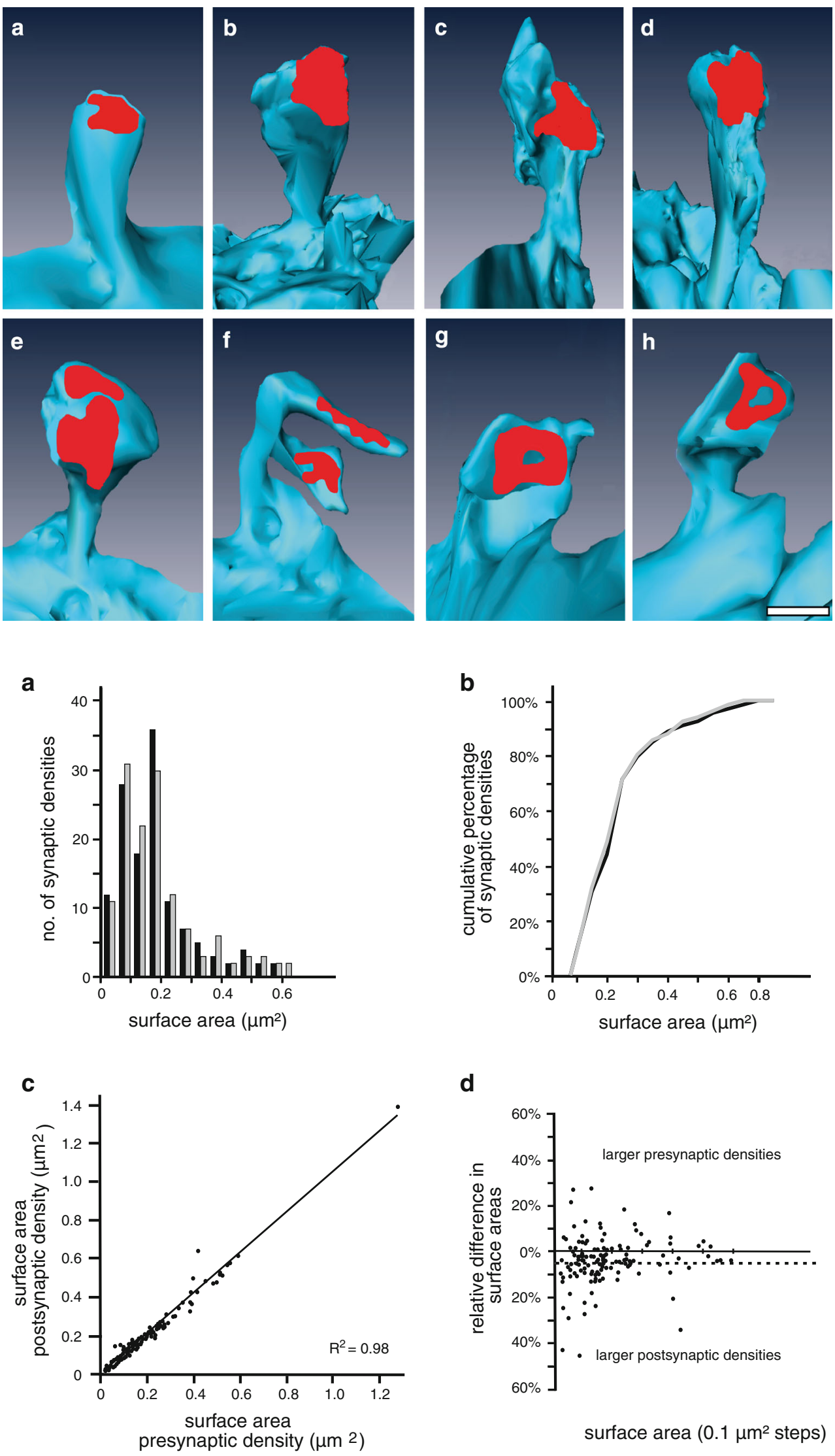

d

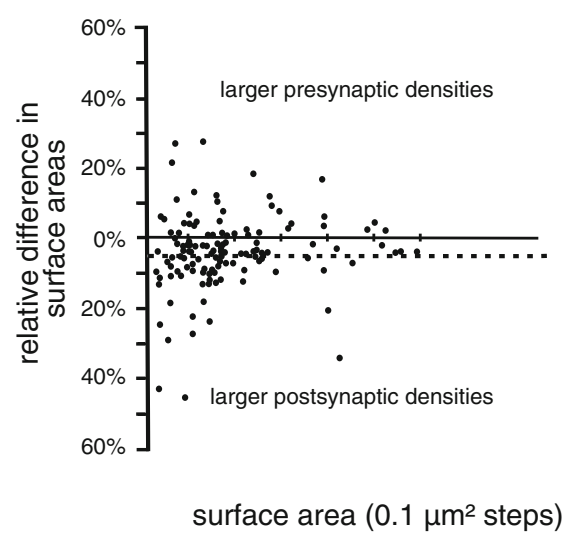



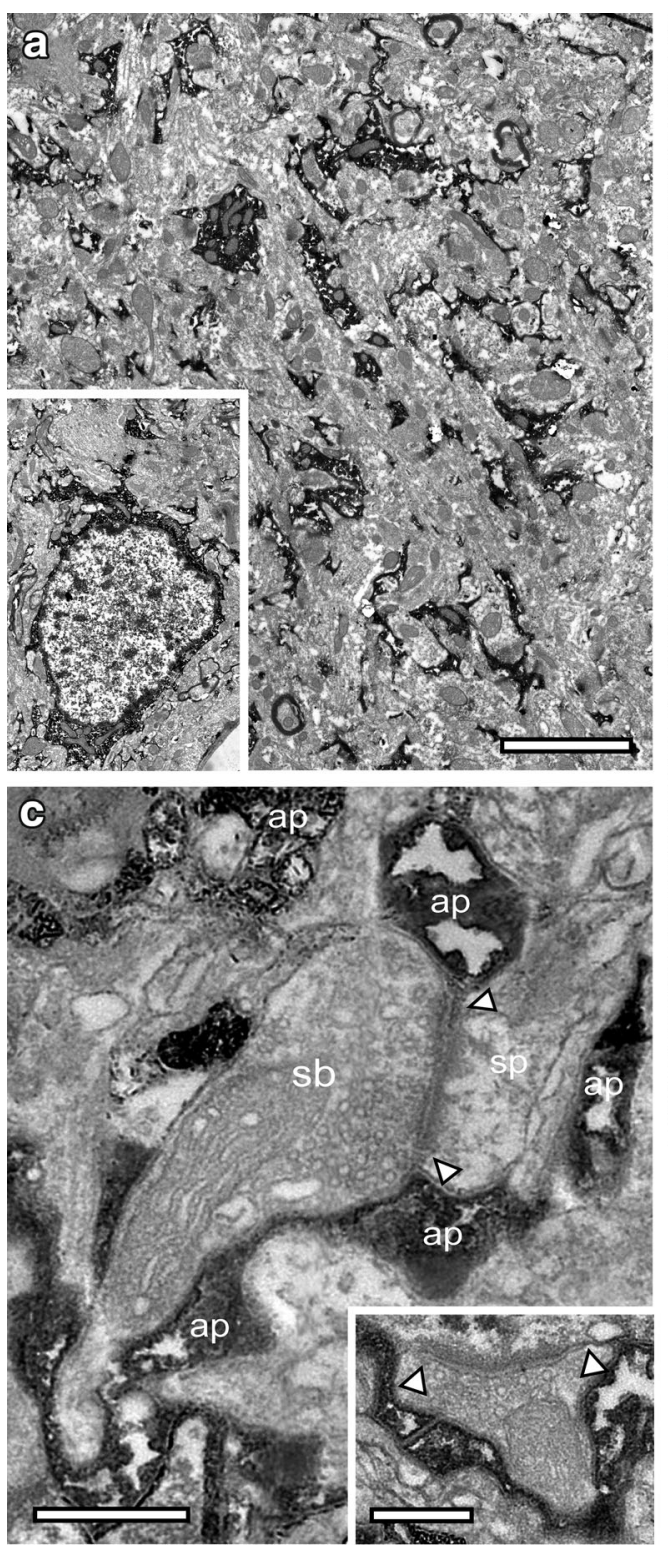

Fig. 8 Astrocytic coverage of intracortical L4 synaptic boutons. a Low power EM micrograph of the distribution of astrocytes as revealed by glutamine synthetase pre-embedding immunohistochemistry (dark DAB reaction product). Note that astrocytes (inset) form a dense network of fine astrocytic processes within layer 4. Scale bar $2 \mu \mathrm{m}$. b Higher order astrocytic segment closely ensheathing several synaptic complexes composed of an individual synaptic bouton (transparent yellow) and its target structure, a spine or dendrite (transparent blue). Scale bar $0.5 \mu \mathrm{m}$. c Electron micrograph of a synaptic complex between a synaptic bouton $(s b)$ and a dendritic

h), thin (Figs. 4a2, 6e) and filopodial-like (Fig. 6f) types. However, the majority $(\sim 75 \%)$ of synaptic boutons was found on stubby and mushroom spines. Furthermore, in the majority of spines $(\sim 80 \%)$ a spine apparatus (Fig. 5 b), a specialized form of the endoplasmic reticulum, was found. It has been hypothesized, that spines containing a spine apparatus are more mobile than those lacking this structural
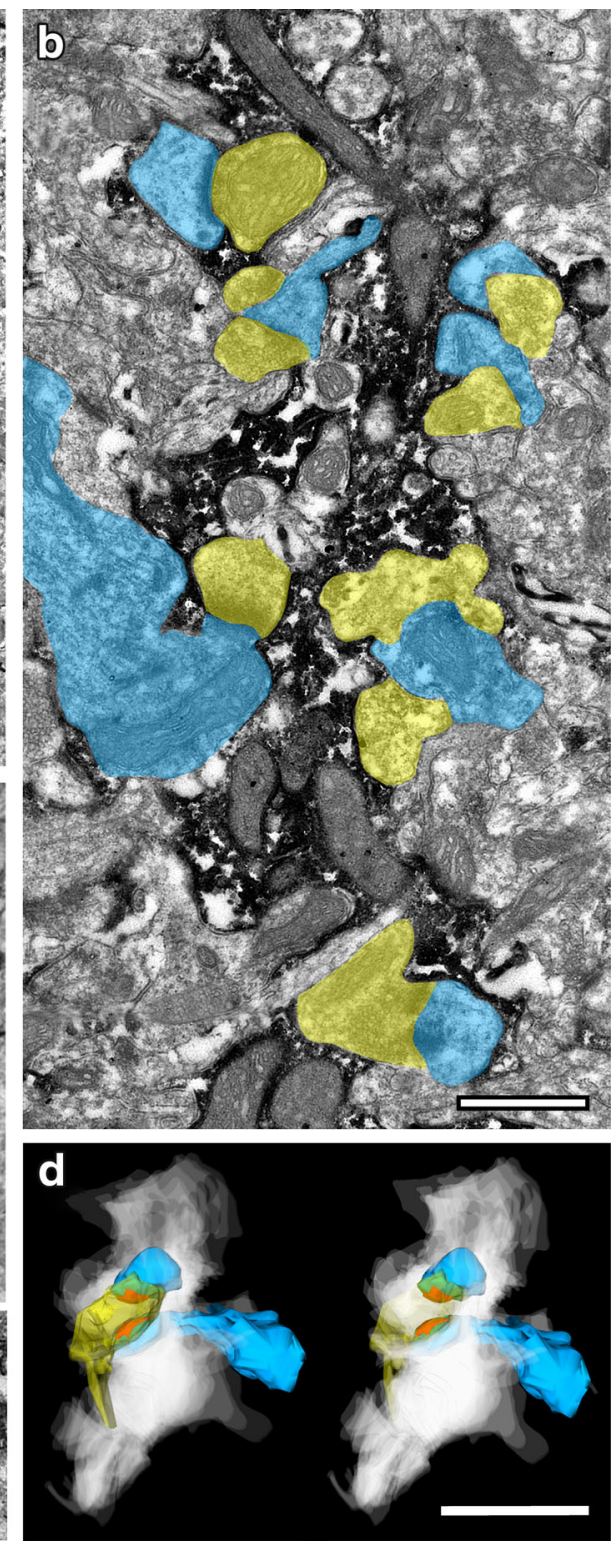

spine $(s p)$ densely covered by fine astrocytic processes (ap). Note that the fine astrocytic processes reach as far as the synaptic cleft at the AZ (arrowheads). Scale bar $0.5 \mu \mathrm{m}$. Inset synaptic bouton establishing a synaptic contact with a dendritic shaft completely ensheathed by fine astrocytic processes. The AZ is indicated by arrowheads. Scale bar $0.25 \mu \mathrm{m}$. d 3D-volume reconstruction of two dendritic spines (blue) and an en passant synaptic bouton (transparent yellow) completely surrounded by fine glial processes (white) viewed in different rotations. Scale bar $2 \mu \mathrm{m}$

subelement and are thought to modulate short- and longterm plasticity (Gray 1959a, b; Deller et al. 2003; Konur and Yuste 2004; Holtmaat et al. 2005; Umeda et al. 2005).

The majority of dendrites receive relatively dense synaptic input (Fig. 4a1-a4, b). For example, 28 synaptic boutons were found at the dendritic segment $(\sim 20 \mu \mathrm{m}$ in length) shown in Fig. 4b. However, dendritic segments 

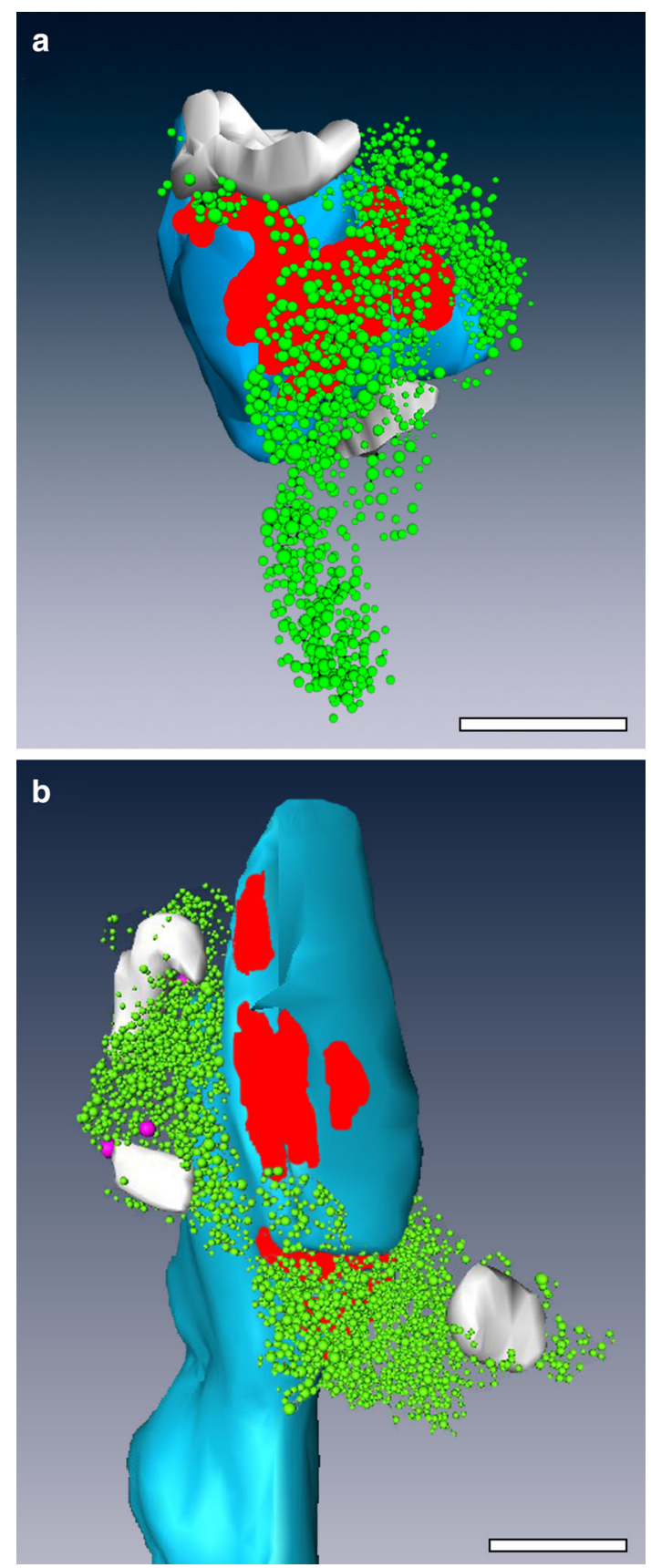

Fig. 9 3D-volume reconstructions of two spine synaptic boutons. a Total pool of synaptic vesicles (green dots) distributed over the entire volume of an endterminal synaptic bouton on a spine head (blue) with a single, but large AZ (red) and two mitochondria (white). Scale bar $0.25 \mu \mathrm{m}$. b En passant synaptic bouton terminating on both the spine neck and head. Note the large pool of synaptic vesicles (green dots) shared by three perforated AZs (red) and mitochondria (white) associated with the pool of synaptic vesicles. Scale bar $0.5 \mu \mathrm{m}$

with a much smaller number of boutons were also observed (Fig. 4c; 9 synaptic boutons on a $\sim 14 \mu \mathrm{m}$ long dendritic segment). Occasionally, individual synaptic boutons ( $\sim 5 \%$ of the total) were seen to establish multiple synaptic contacts with two or three spines (Fig. 5f) on either the same or different dendrites. The majority of synaptic boutons were excitatory $(\sim 85 \%)$, located predominantly on spines, and occasionally on shafts. The remaining $\sim 15 \%$ were inhibitory synaptic boutons as revealed by GABA postembedding immunohistochemistry (Fig. 3e). Interestingly, so-called dendro-dendritic synapses (Fig. 5g) were infrequently observed.

Intracortical L4 synaptic boutons varied in size, shape and vesicle numbers (Tables 1,2 ). Their mean surface area was $3.03 \pm 0.71 \mu \mathrm{m}^{2}$ (min: $0.46 \mu \mathrm{m}^{2}$; $\max : 12.38 \mu \mathrm{m}^{2}$ ) with a volume of $0.20 \pm 0.07 \mu \mathrm{m}^{3}$ (min: $0.01 \mu \mathrm{m}^{3}$; $\max$ : $\left.1.36 \mu \mathrm{m}^{3}\right)$; both values were highly correlated $\left(r_{\mathrm{s}} 0.94\right)$. Only slight inter-individual statistical differences were observed for both parameters within the group of experimental animals (Suppl. Fig. 1A-C). In larger synaptic boutons, two or three, often quite large mitochondria were found, whereas small boutons contained only a single or no mitochondrion (Figs. 5a, b, e, f, 9). When present, mitochondria contributed on average $\sim 20 \%$ to the total volume of synaptic boutons (see also Table 1). The volume of mitochondria differed significantly $(p=0.05)$ between experimental animals (Suppl. Fig. 1D). Interestingly, their volume was well correlated with the volume of boutons (Fig. 11a), but only slightly correlated with the total number of synaptic vesicles as indicated by the correlation factors of 0.95 and 0.46 , respectively. This suggests a direct relationship between the size of the synaptic bouton with the size of mitochondria, but not with the pool of synaptic vesicles. However, both correlations may partially contribute to the mode and machinery of release at L4 synaptic boutons (see "Discussion").

Furthermore, we observed so-called multivesicular bodies, i.e., endosomal organelles containing several internal vesicles involved in endocytotic and trafficking functions in $\sim 15 \%$ of synaptic boutons (not shown).

Structural composition of AZs at the preand postsynaptic apposition zone

The shape and size of the $\mathrm{AZ}$ are key structural elements involved in the induction, maintenance and termination of synaptic transmission, as well as in the regulation of synaptic plasticity. Per definition, an AZ, the structural equivalent to a functional transmitter release site, is characterized by the typical broadening of the synaptic cleft, the symmetric or asymmetric pre- and postsynaptic densities, and the accumulation of synaptic vesicles located close to the presynaptic density (Fig. 5). In L4 synaptic boutons, several synaptic vesicles (2-13) were found which were closely attached or fused (docked) with the presynaptic density (Fig. 5c), pointing to multivesicular release at these synapses. The majority of intracortical L4 synaptic 

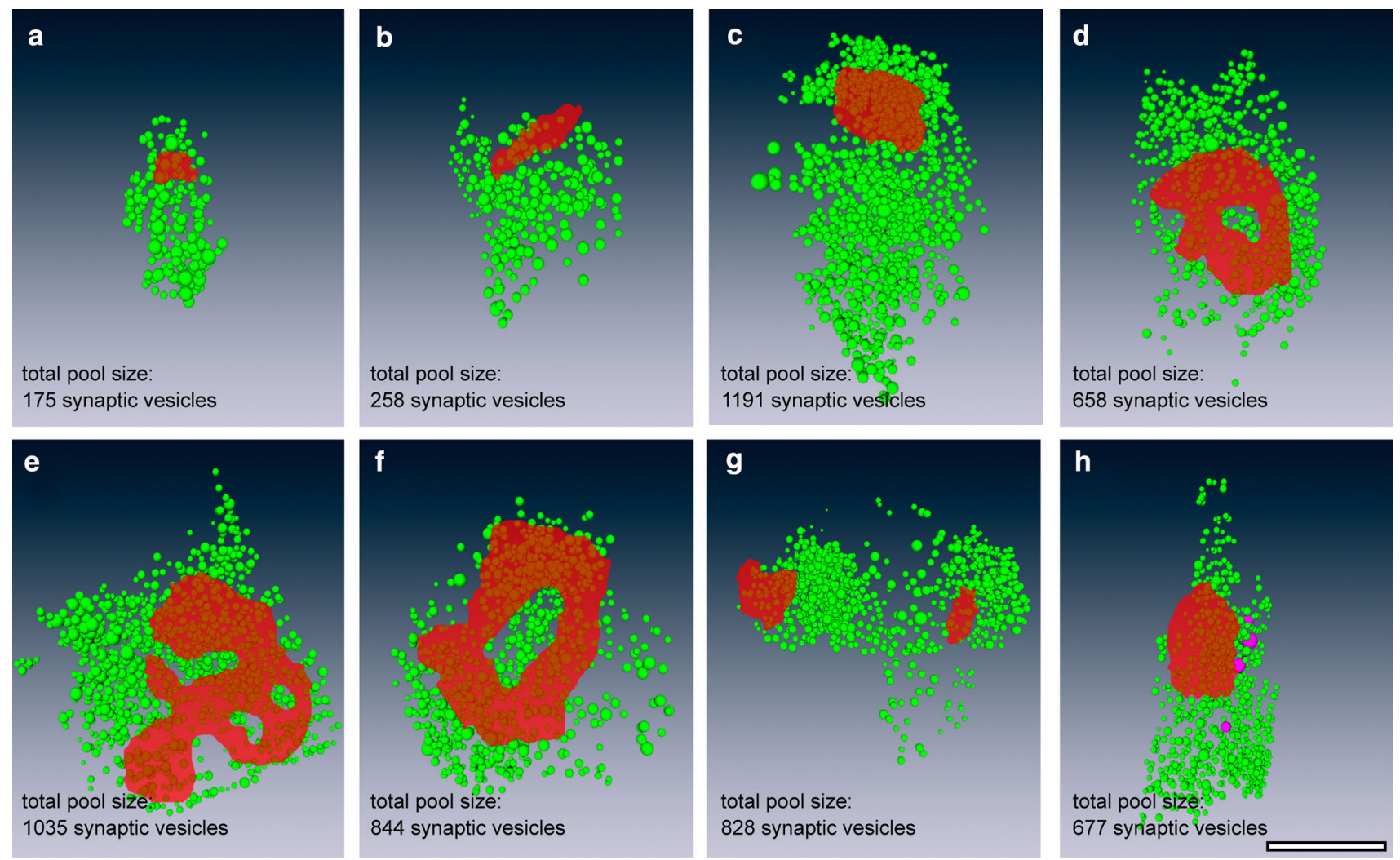

Fig. 10 Comparison of the size of the total pool of synaptic vesicles between individual intracortical L4 synaptic boutons. $\mathbf{a}-\mathbf{h}$ 3D-volume reconstructions of individual total pools of synaptic vesicles (green dots) at AZs (given in red). Note the large differences in the total pool size of synaptic vesicles. At some boutons, the pool of synaptic

boutons $(\sim 95 \%)$ contained only a single AZ. The remainder had two or at the most three AZs (Figs. 5a-d, 9a; see also "Discussion").

The pre- and postsynaptic densities formed bands of electron-dense fuzzy material, $\sim 73 \%$ showed perforations at either the pre- or apposing postsynaptic membrane, or at both membranes (Figs. 5a, c, d, e, 6g, h). Notably, $\sim 35 \%$ of the AZs showed perforations of the postsynaptic density (Fig. 5a, e); the remainder had perforations in the presynaptic density, or were non-perforated (Figs. 5a, b, f, $\mathrm{g}, 6 \mathrm{a}-\mathrm{f}$ ). Perforated postsynaptic densities occurred predominantly on axo-spinous rather than on axo-dendritic synapses $(>80 \%)$. It has been hypothesized that the perforation of a postsynaptic density is one structural correlate of high synaptic efficacy (Peters and Kaiserman-Abramof 1969; Geinisman et al. 1991, 1993; Nicoletta et al. 2013).

Size of pre- and postsynaptic densities at the apposition zone

The mean surface area of AZs was estimated separately for pre- and postsynaptic densities, and was on average $0.18 \pm 0.06 \mu \mathrm{m}^{2}$ for either membrane specialization

vesicles is shared between individual AZs (g). Large dense-core vesicles (magenta dots in $\mathbf{h}$ ) were frequently observed. No cluster-like arrangements of individual pools of synaptic vesicles around individual AZs were found. Scale bar $0.5 \mu \mathrm{m}$

(Fig. 7a; see also Table 1). We found both very large [1.28 $\mu^{2}$ (pre), $1.39 \mu \mathrm{m}^{2}$ (post)], but also very small $\left[0.02 \mu \mathrm{m}^{2}\right.$ (pre and post)] pre- and postsynaptic densities, with a skew towards smaller AZs (Fig. 7a, b). In $\sim 85 \%$ of the examined AZs, we found a nearly perfect overlap in size and position of the pre- and postsynaptic densities at the apposition zone regardless of their size (Fig. 7c), as indicated by both the $R^{2}$ (see Fig. 7c) and $r_{\mathrm{s}}$ value (0.98). In the remaining $\sim 15 \%$ of AZs, either the preor the postsynaptic density was slightly larger with a skew to larger postsynaptic densities (Fig. 7d). The surface area of the presynaptic densities was slightly correlated $\left(r_{\mathrm{s}} 0.52\right)$ with that of synaptic boutons, suggesting that the size of the AZ is independent from that of the synaptic bouton (Fig. 11b). In two out of five animals, a significant difference $(p \leq 0.05)$ in surface area of the two membrane specializations was found (Suppl. Fig. 2A).

The average cleft diameter at AZs measured under the pre- and postsynaptic densities was $17.22 \pm 1.50 \mathrm{~nm}$ at the lateral edges and $30.22 \pm 1.42 \mathrm{~nm}$ in the central region (Table 1). Notably, no difference for the cleft width was found between small and larger AZs. 
So-called puncta adhaerentia, adhesion complexes at the pre- and postsynaptic apposition zone, as observed at the calyx of Held endterminal (Sätzler et al. 2002), the hippocampal (Rollenhagen et al. 2007) and the cerebellar MFB (Xu-Friedman et al. 2001) were not found at intracortical L4 synaptic boutons.

In summary, the shape and size of the pre- and postsynaptic densities constituting the AZ at dendritic spines and shafts, and the occurrence of a spine apparatus may contribute to, and thus partially explain, the high synaptic efficacy and strength at spine synaptic boutons of excitatory L4 neurons (see Feldmeyer et al. 1999).

Isolation of individual L4 synaptic boutons by fine astrocytic processes

Glial cells, in particular astrocytes and their fine processes, have been shown to play an important role in synaptic behavior due to their active removal of excessive or 'spilled' glutamate from the synaptic cleft. Furthermore, they structurally isolate synaptic contacts, thereby modulating synaptic transmission and plasticity (Brückner et al. 1993; Oliet et al. 2004; Houades et al. 2008; for review see Danbolt 2001).

Pre-embedding immunohistochemistry against glutamine synthetase, a key enzyme expressed in astrocytes, was carried out to examine the relationship between individual L4 synaptic boutons and astrocytes, in particular their fine processes (Fig. 8). In two large series of ultrathin sections (each comprising $\sim 150$ sections), astrocytic processes, synaptic boutons and their postsynaptic target dendrites or spines were analyzed, some of which were three-dimensionally reconstructed (Fig. 8). EM examination revealed that astrocytes and their fine processes formed a dense network within individual 'barrels' in layer 4 (Fig. 8a). In the majority of cases $(>90 \%)$, individual synaptic boutons and their target structures, forming individual synaptic complexes, were completely surrounded by fine astrocytic processes (Fig. 8b, c). Individual astrocytic segments, even of higher order, were seen to tightly enwrap multiple synaptic complexes, isolating them from the surrounding neuropil and from neighboring synaptic complexes (Fig. 8b). Astrocytic fingers were observed to reach as far as to individual AZs, or even the synaptic cleft (Fig. 8b-d). Thus, they are in a key position to regulate the temporal and spatial concentration of glutamate and prevent glutamate spillover by controlling and terminating the extent of vertical and horizontal diffusion of glutamate in the synaptic cleft (see "Discussion").

Size and organization of the pool of synaptic vesicles

Another key determinant for synaptic efficacy, strength, reliability and mode of release is the organization of the pool of synaptic vesicles. In particular, the size of the functionally defined readily releasable (RRP), the recycling (RP) and reserve pool as measured by imaging, capacitance measurements, quantal analysis studies, or electron tomography is a critical factor controlling and regulating synaptic transmission and plasticity (Sahara and Takahashi 2001; Hallermann et al. 2003; Silver et al. 2003; Saviane and Silver 2006; Watanabe et al. 2013; reviewed by Schneggenburger et al. 2002; Rizzoli and Betz 2005).

Synaptic vesicles were distributed throughout the entire synaptic bouton and partially also found in the axon, and were often densely packed (Figs. 5, 9, 10). They occupied on average $\sim 5 \%\left(0.01 \mu \mathrm{m}^{3}\right)$ of the total volume of the synaptic bouton (Table 2). Strikingly, the total pool size varied substantially ranging from 38 to 1,784 with a mean of $561 \pm 108$ vesicles per synaptic bouton (Fig. 10; see Table 2). This variability may contribute to the experimentally observed variance in unitary EPSP amplitudes among L4 excitatory connections (Feldmeyer et al. 1999, 2002, 2005; Silver et al. 2003).

Only a weak correlation between the total pool of synaptic vesicles with the total surface area $\left(r_{\mathrm{s}} 0.57\right.$, Fig. 11c), the total volume of synaptic boutons $\left(r_{\mathrm{s}} 0.62\right.$, Fig. 11d) and the surface area of presynaptic densities $\left(r_{\mathrm{s}} 0.53\right.$, Fig. 11e) were found. Hence, the total pool size seems to be independently regulated suggesting differences in synaptic efficacy and strength at individual synaptic boutons. No significant differences between the experimental animals were observed (Suppl. Fig. 2B).

Synaptic vesicles had a mean diameter of $29.85 \pm 4.63 \mathrm{~nm}$ ranging from 18.39 to $48.67 \mathrm{~nm}$. No significant difference was found for the vesicle diameter between the three different pools of synaptic vesicles $(p>0.05)$. However, a slight but significant difference in this parameter was found between synaptic boutons at biocytin-filled neurons and that from the other experimental animals (see Table 2; Suppl. Fig. 2C).

In addition to small clear vesicles, large dense-core vesicles were found in $\sim 50 \%$ of intracortical L4 synaptic boutons (Fig. 9b) ranging from 48 to $72 \mathrm{~nm}$ with an average diameter of $62.46 \pm 5.05 \mathrm{~nm}$. Dense-core vesicles were often seen to fuse either with the presynaptic membrane at extrasynaptic locations of the bouton, or at the presynaptic density. It is therefore conceivable that besides being involved in endo- and exocytosis (Watanabe et al. 2013), dense-core vesicles also contribute to the build-up and organization of AZs by, for example, releasing piccolo and bassoon (Shapira et al. 2003; Schoch and Gundelfinger 2006), or by clustering synaptic vesicles at the presynaptic density (Mukherjee et al. 2010; Watanabe et al. 2013; for review see Südhof 2012). 

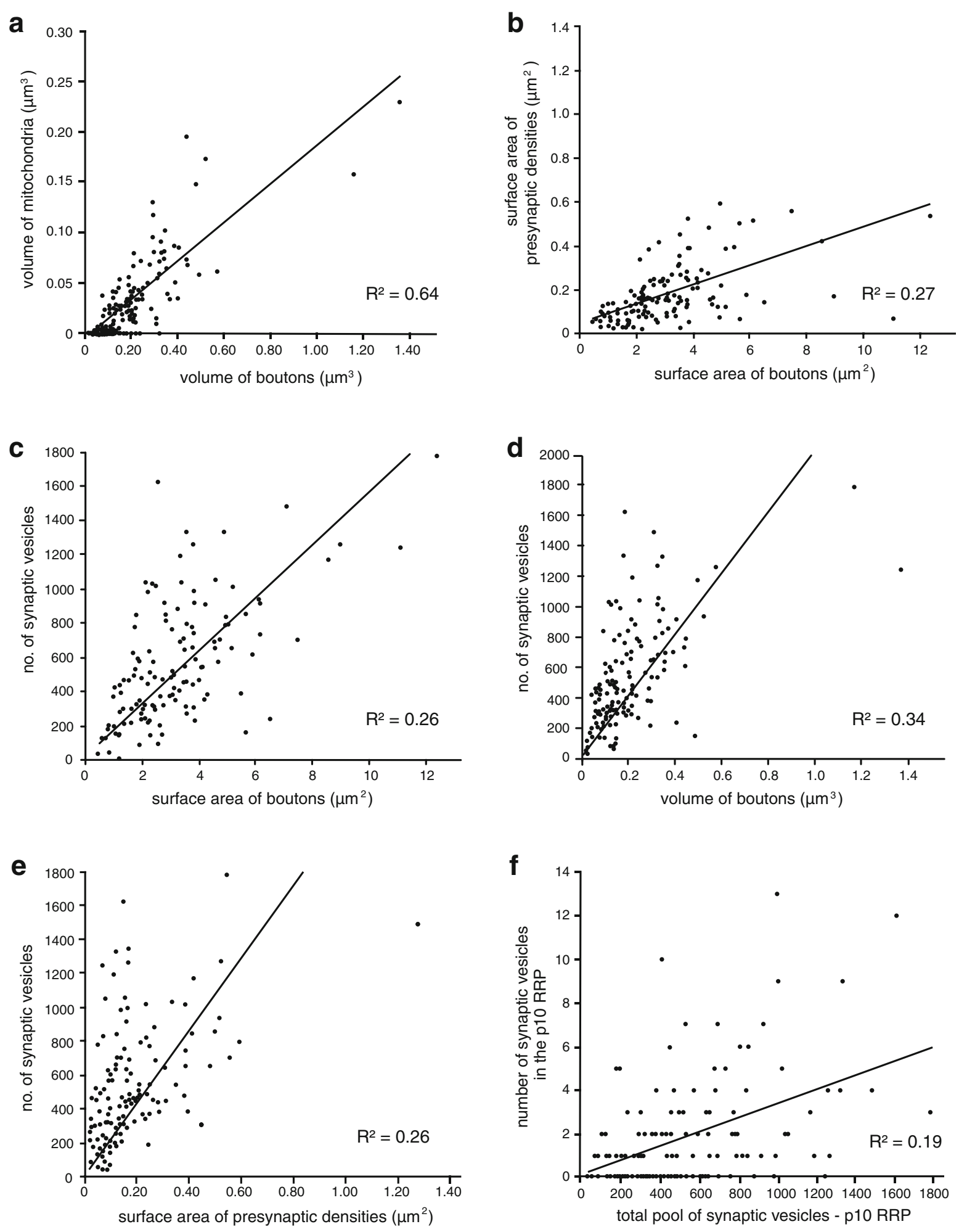

Fig. 11 Correlation of various structural parameters of L4 synaptic boutons. Between a the volume of boutons vs. volume of mitochondria, $\mathbf{b}$ the surface area of boutons vs. surface area of the presynaptic densities, $\mathbf{c}$ the surface area of boutons vs. total number of synaptic vesicles, d the volume of boutons vs. total number of synaptic

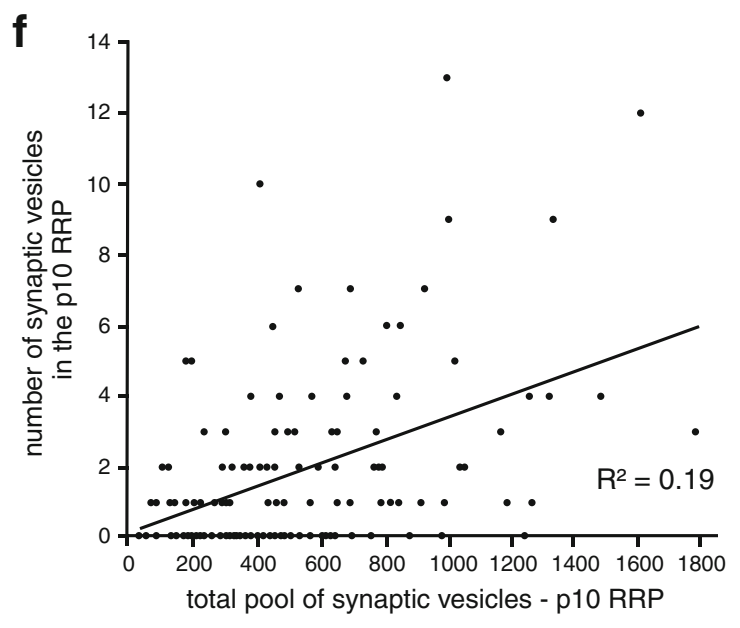

vesicles, $\mathbf{e}$ the surface area of presynaptic densities vs. total number of synaptic vesicles, $\mathbf{f}$ the total pool of synaptic vesicles minus the RRP within a perimeter of $10 \mathrm{~nm}$ vs. number of synaptic vesicles within the RRP at the $10 \mathrm{~nm}$ perimeter. Data points were all fitted for linear regression and the $R^{2}$ is given for each correlation 
a

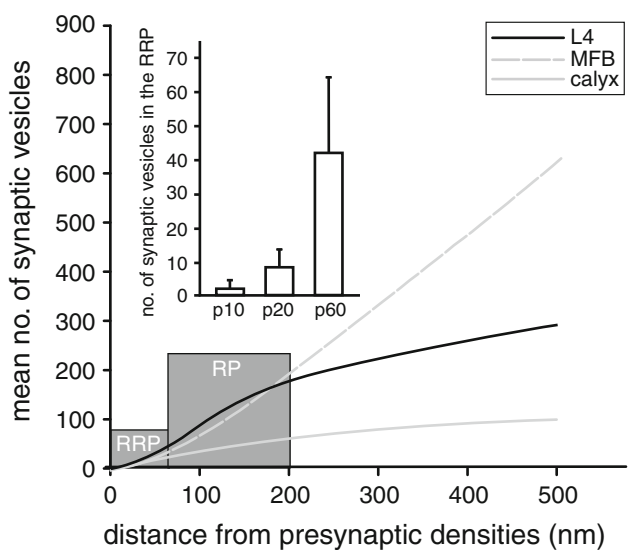

b

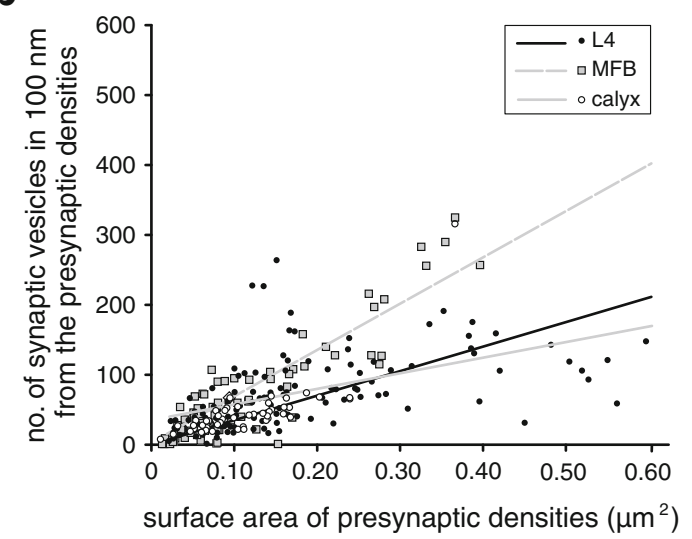

Fig. 12 Number of synaptic vesicles per synaptic bouton and at individual AZs. a The graph shows the correlation for the mean number of synaptic vesicles as a function of the distance from a presynaptic density (averaged over all presynaptic densities) for L4 synaptic boutons (black line), MFBs (dashed gray line) and the calyx of Held (solid gray line). The gray boxes indicate the borders between the respective RRP, the RP and the reserve pool of synaptic vesicles. Note that the slope of the curves is $\sim$ threefold larger for L4 synaptic boutons and MFBs than that of the calyx of Held endterminal. Inset mean number $\pm \mathrm{SD}$ of synaptic vesicles within a perimeter of 10,20 , and $60 \mathrm{~nm}$ from the presynaptic density. Note the large increase of the RRP by $\sim$ threefold and 15 -fold between the three perimeters. b Relationship between the number of synaptic vesicles located within a perimeter of $100 \mathrm{~nm}$ from the presynaptic density representing vesicles in the putative RP and the surface area of individual presynaptic densities for L4 synaptic boutons (black dots), adult MFBs (gray squares; Rollenhagen et al. 2007) and the calyx of Held endterminal (open dots; Sätzler et al. 2002). Data points were fitted by linear regression. Note that for MFBs the slope is much larger when compared with the calyx of Held and L4 synaptic boutons. Corresponding squares of $R^{2}$ were 0.31 for L4 synaptic boutons, 0.82 for MFBs and 0.59 for the calyx of Held, respectively

Readily releasable, recycling and reserve pools

We attempted to identify subsets of synaptic vesicles as structural correlates for the three functionally defined pools of releasable quanta of neurotransmitters as postulated from measurements of release rates. Since morphologically no clear distinction between the three functionally defined pools can be made (Figs. 5, 9, 10), the RRP, the RP and the reserve pools were tentatively defined using the following criteria: vesicles within a perimeter of $0-60 \mathrm{~nm}$ (Rizzoli and Betz 2005; but see also Watanabe et al. 2013) from the presynaptic density constituted the putative RRP (Fig. 12a), those within a $60-200 \mathrm{~nm}$ perimeter ( $\sim 5$ vesicle diameters) were considered to be the putative RP (Fig. 12a, b), and those at a distance $>200 \mathrm{~nm}$ were classified as belonging to the reserve pool (Fig. 12a; Table 2).

The average number of synaptic vesicles located within a perimeter of $10 \mathrm{~nm}$ from the presynaptic density, representing the equivalent to 'docked' vesicles, was $1.97 \pm 2.57$ (min. 2, max. 13). At the $20 \mathrm{~nm}$ perimeter, this number increased to $6.30 \pm 6.40$ (equivalent to tethered vesicles; Watanabe et al. 2013) although a huge variability was found as also indicated by the range ( $\min .3$, max. 33). At the $60 \mathrm{~nm}$ perimeter a more than sixfold increase to $38.60 \pm 7.37$ (min. 3, max. 135) was measured, equivalent to $6.8 \%$ of the total pool of synaptic vesicles (see also inset Fig. 12a). The steep increase in the number of vesicles within the $10-60 \mathrm{~nm}$ perimeter raises the question whether, for example, the $10 \mathrm{~nm}$ RRP ('docked' vesicles) is directly correlated with the total pool size. Interestingly, only a slight correlation $\left(r_{\mathrm{s}} 0.44\right)$ was found (Fig. 11f), although for the 20 and $60 \mathrm{~nm}$ perimeters the $r_{\mathrm{s}}$ increased to 0.49 and 0.60 , respectively.

The putative RP contained between 18 and 633 vesicles with a mean of 130.16 , representing $\sim 23 \%$ of the total pool (see Table 2). A comparison of the RP in different types of synapses, and taken the $100 \mathrm{~nm}$ perimeter, showed that although L4 synaptic boutons belong to the smaller CNS synapses, their RP is as large as that of the MFB, and even larger than that of the calyx of Held-principal neuron endterminal (Fig. 12). However, the RP also showed a large variability, as indicated by the standard deviation and the large range in pool size.

The remaining synaptic vesicles belong to the putative reserve pool, with an average size of 408.84 synaptic vesicles ( $\sim 70 \%$ of the total pool).

It should be noted that no significant differences for the four perimeters measured were found between the experimental groups of animals (Suppl. Fig. 3).

\section{Discussion}

This is the first quantitative analysis of a cortical synapse as exemplified by synaptic boutons terminating on L4 excitatory spiny neurons in the 'barrel field' of the rat somatosensory cortex. L4 synaptic boutons are small in surface area and volume compared to other CNS synapses, although their AZ area, covering both the pre- and 
postsynaptic density, is comparably large and their vesicular content in the three pools is high. These findings may underlie the high release probability and thus synaptic efficacy observed for L4 excitatory connections. Additionally, the broad distribution of AZ size and numbers of synaptic vesicles also reflects the wide range in release probability at individual synapses in a given connection (Feldmeyer et al. 1999). In addition, the comparatively large putative RRP, RP, and reserve pools, as well as the astrocytic enwrapping, are likely to contribute to the ability of these synapses to maintain high release rates during repetitive stimulation (Feldmeyer et al. 1999, 2002, 2005; Barth 2002).

Shape and size of AZs and other structural features and release probability

It has recently been shown that both the size and shape of the $\mathrm{AZ}$ are the major driving forces for the reliability, RRP size and release probability $\left(p_{\mathrm{r}}\right)$ at individual synaptic contacts in the hippocampal CA1 subregion (Matz et al. 2010; Freche et al. 2011; Holderith et al. 2012). There, large AZs have a much higher $p_{\mathrm{r}}$, RRP and a higher number of presynaptic $\mathrm{Ca}^{2+}$ channels when compared with smaller AZs (Holderith et al. 2012). Moreover, the geometry of the AZs is regulated in an activity-dependent manner; a change in their geometry and size can occur within minutes after the onset of stimulation (Matz et al. 2010).

Furthermore, it has been hypothesized that perforations of postsynaptic densities are another structural correlate of high synaptic efficacy (Geinisman et al. 1991, 1993). Recently, a direct correlation between AZ area and perforated postsynaptic densities with the number of 'docked' and reserve pool vesicles was found. The total number of 'docked' and reserve pool vesicles in perforated postsynaptic densities significantly exceeded that in non-perforated postsynaptic densities(Nicoletta et al. 2013).

The surface area of both the pre- and postsynaptic densities at L4 synaptic boutons was $0.18 \pm 0.06 \mu \mathrm{m}^{2}$, although a large variability $(\mathrm{CV}=0.84)$ in size with a skew to smaller AZs was found. However, this large variability in AZ size was also described for other synapses in the CNS (for review see Xu-Friedman and Regehr 2004; Rollenhagen and Lübke 2006). Notably, AZs investigated here were on average $\sim 2$ - to 3-fold larger in surface area when compared with other, even much larger CNS terminals. This may partially contribute to quantal variability and also determine the mode of release. In addition, the wide range in the size and distribution of AZs together with a large variability in spine size and morphology may also contribute to the high degree in synaptic plasticity observed at intracortical L4 synaptic boutons, in particular at axospinous synapses. Thus, the comparatively large AZs together with the high proportion of perforated postsynaptic densities $(\sim 35 \%)$ are likely to represent a key structural element for the high synaptic efficacy, strength and plasticity of excitatory connections of L4 neurons (Feldmeyer et al. 1999, 2002, 2005).

The geometry of the AZs, the number of 'docked' vesicles (2-13 taken the p10 perimeter) together with a relatively small cleft diameter $(\sim 20 \mathrm{~nm})$ allows a rapid diffusion and binding of glutamate to the main excitatory receptors in the neocortex, namely to AMPA and NMDA receptors (Feldmeyer et al. 1999; Rollenhagen et al. 2012). Thus, one would predict that the structural geometry of the AZs is a pre-requisite for synapses with a high $p_{\mathrm{r}}$ and RRP, which is indeed the case in young adult L4-L4 excitatory connections (Feldmeyer et al. 1999). This is further substantiated by the fact that this connection is strong enough to generate suprathreshold postsynaptic APs, a phenomenon never observed in other intra- and translaminar cortical connections. On the other hand, although the size of AZs and the RRP is relatively large, a frequent fluctuation in the unitary EPSP amplitude, failure rate, coefficient of variation and paired-pulse depression is observed at L4-L4 excitatory connections (Feldmeyer et al. 1999; this study), which could be explained by the huge variability in pool sizes between individual synaptic boutons within a given connection.

Finally, it has been reported that mitochondria act not only as internal calcium stores (Pozzan et al. 2000; Rizzuto et al. 2000), but may also regulate and adjust internal $\mathrm{Ca}^{2+}$. levels in synapses. Furthermore, mitochondria are highly mobile (Mironov 2006; Mironov and Symonchuk 2006), and may be critically involved in the mobilization of synaptic vesicles from the reserve pool (Verstreken et al. 2005). This could explain why in L4 synaptic boutons mitochondria, when present, were always closely associated with the pool of synaptic vesicles (Figs. 4, 7, 9).

Altogether, the structural variability as indicated by the geometry of L4 synaptic boutons, the presence of mitochondria, and the size and shape of the AZs strongly suggest that synaptic reliability, strength and plasticity are governed and modulated individually at excitatory L4 synaptic boutons.

\section{Glial coverage of L4 synaptic boutons}

Another striking difference to other CNS synapses was the astrocytic ensheathment of intracortical L4 synaptic boutons. Here, astrocytes were spatially organized within 'barrels', but not in the septa, favoring communication within a 'barrel' rather than signaling between two neighboring 'barrels' (Houades et al. 2008; this paper). In addition, it was reported that synapses with a higher number of 'docked' vesicles are almost exclusively 
ensheathed by astrocytic processes. Hence, it has been hypothesized that the degree of astrocytic ensheathment correlates with the activity of a synapse (Brückner et al. 1993). Furthermore, astrocytes also release transmitters, for example glutamate and GABA (Le Meur et al. 2012), through vesicular exocytosis that also can interfere with, and control, the release machinery of synapses. Hence, astrocytes can modulate synaptic transmission through the activation or inhibition of pre- and postsynaptic receptors (Haydon and Carmignoto 2006). A study by Min and Nevian (2012) has shown that astrocytes are crucial for the induction and control of spike time-dependent depression (t-LTD) at neocortical synapses by gradually increasing their $\mathrm{Ca}^{2+}$ signaling during the induction of t-LTD in layers 4 and $2 / 3$. Thus, astrocytes may act as a memory buffer for previous coincident neuronal activity and are therefore involved in modulating synaptic transmission and plasticity at intracortical L4 synaptic boutons. Recently, it has been shown in the hippocampal CA1 subregion, that Connexin 30, one of the two major gap-junctional proteins, modulates excitatory synaptic transmission by controlling the extension of astrocytic processes into the synaptic cleft, thus regulating the glutamate clearance through a nonchannel function. The absence of connexin30 in connexin $30^{-1-}$ mice is associated with an increased glutamate clearance and, consequently, reduced glutamate transmission (Pannasch et al. 2014).

The majority of intracortical L4 synaptic boutons and their target structures $(\sim 80 \%)$ were surrounded and tightly ensheathed by fine astrocytic processes thereby isolating neighboring synaptic complexes. Furthermore, fine astrocytic processes reach as far as the synaptic cleft. Hence, they may act as physical barriers to neurotransmitter diffusion, thereby preventing spillover of horizontally diffusing glutamate. Glutamate transporters enable astrocytes to actively take-up excessive or 'spilled' glutamate and, therefore, aid to terminate synaptic transmission and speed-up the recovery from receptor desensitization as described for other synapses (Oliet et al. 2004; for review see Danbolt 2001). Both mechanisms enable the precise spatial and temporal regulation of the concentration of the neurotransmitter in the synaptic cleft at these synapses. Given the tight coverage of synaptic complexes in layer 4, these mechanisms are likely to occur also at L4 synaptic boutons, which is another add-on mechanism in shaping the unitary EPSP amplitude.

This tight astrocytic ensheathing of L4 synaptic boutons is in marked contrast to findings at the hippocampal MFBCA3 pyramidal cell synapse (Rollenhagen et al. 2007) and the calyx of Held-principal neuron synapse in the medial nucleus of the trapezoid body (Müller et al. 2009). At MFBs synaptic boutons, astrocytic processes were never located close to individual AZs, but rather ensheathed individual synaptic complexes from the surrounding neuropil. This may explain the occurrence of glutamate spillover and, as a consequence, synaptic cross talk and the switch from asynchronous to synchronous release upon repetitive stimulation, which has been observed at both synapses (Hallermann et al. 2003; Rodríguez-Contreras et al. 2008; for review see von Gersdorff and Borst 2002).

Thus, the contribution of astrocytes to synaptic transmission and plasticity seems to be individually regulated at CNS synapses and in different brain regions.

Size and organization of the three distinct pools of synaptic vesicles

The size of the three functionally defined pools of synaptic vesicles, in particular the RRP and RP are one of the most important structural parameters in synaptic transmission and plasticity. We made an attempt to estimate a putative structural correlate for the three pools according to published criteria (Rosenmund and Stevens 1996; Schikorski and Stevens 2001; Rizzoli and Betz 2004; Watanabe et al. 2013; for review Rizzoli and Betz 2005). The total pool of synaptic vesicles comprises only a small fraction $(\sim 5 \%)$ of the total volume of L4 synaptic boutons with a total pool size of $561 \pm 108$ synaptic vesicles at a single AZ. This size is comparable with that found in other much larger CNS synapses (see Table 2 in Rollenhagen and Lübke 2006). The calyx of Held endterminal contains $\sim 70,000$ vesicles distributed over $\sim 550 \mathrm{AZs}$, resulting in $\sim 125$ synaptic vesicles/AZ (Sätzler et al. 2002). In adult MFBs, a total pool size of $\sim 16,000$ was estimated for on average 20 $\mathrm{AZs}$, leading to $\sim 900$ synaptic vesicles/AZ (Rollenhagen et al. 2007). At cerebellar mossy fiber terminals, a total pool size of 200,000 synaptic vesicles at $\sim 330$ release sites was estimated (Saviane and Silver 2006) with $\sim 600$ synaptic vesicles/AZ. Although L4 synaptic boutons are the smallest in surface area $\left(\sim 3 \mu \mathrm{m}^{2}\right)$ compared to hippocampal $\left(\sim 60 \mu \mathrm{m}^{2}\right)$ and cerebellar MFBs $\left(200 \mu \mathrm{m}^{2}\right)$, or to the calyx of Held endterminal $\left(\sim 2,500 \mu \mathrm{m}^{2}\right)$, their total pool size is either comparable with that of other synaptic boutons, or even fivefold larger (calyx of Held endterminal, Fig. 12b).

We subdivided the total pool into vesicles located within a perimeter of $60 \mathrm{~nm}$, that may constitute the putative RRP, a group located between 60 and $200 \mathrm{~nm}$, the putative RP, and vesicles found outside the $200 \mathrm{~nm}$ perimeter, the putative reserve pool (Fig. 12; Rosenmund and Stevens 1996; but see Watanabe et al. 2013; for review see Rizzoli and Betz 2005). Additionally, we defined two subdivisions within the RRP based on the 10 and $20 \mathrm{~nm}$ perimeters.

Hence, the RRP/AZ at L4 synaptic boutons was $\sim 2$, $\sim 6$ and $\sim 40$ synaptic vesicles under the 10, 20 and $60 \mathrm{~nm}$ perimeters, respectively. The total RRP/550AZs at the 
calyx of Held is $\sim 1,100$ vesicles, corresponding to only two 'docked' synaptic vesicles/AZ (Sätzler et al. 2002) at a perimeter of $10 \mathrm{~nm}$. The total RRP was $\sim 2,800$ and $\sim 10,000$ vesicles/550AZs at the 20 and $60 \mathrm{~nm}$ perimeter, or $\sim 5$ and $\sim 18$ synaptic vesicles/AZ, respectively. At adult MFBs, the entire RRP was on average $\sim 600$ synaptic vesicles (range 400-1,000). At a perimeter of $10 \mathrm{~nm} 1.8$ vesicles were estimated, at the $20 \mathrm{~nm}$ perimeter this number increased to 6.3 . On average, $\sim 40$ synaptic vesicles that may constitute the RRP were found within a perimeter of $60 \mathrm{~nm}$ at single AZs, although it is questionable whether vesicles at this perimeter belong to the RRP or the RP (Watanabe et al. 2013).

Interestingly, the RRP at intracortical L4 synaptic boutons was comparable to estimated values in adult MFBs and in the calyx of Held at a 10 and $20 \mathrm{~nm}$ perimeter (Fig. 12a), but exceeds the other CNS boutons when the $60 \mathrm{~nm}$ criterion is applied (see Rizzoli and Betz 2005).

The putative RP in L4 synaptic boutons comprised $\sim 130$ synaptic vesicles $(\sim 23 \%$ of the total pool). For adult MFBs and the calyx of Held, the putative RP was $\sim 3,200$ vesicles and $\sim 25,000$, and thus $\sim 175$ and $\sim 45$ synaptic vesicles/AZ, respectively. Hence, the RP at L4 synaptic boutons was $\sim$ threefold larger than that at the calyx of Held (Fig. 12a).

Finally, the putative reserve pool was $\sim 400$ synaptic vesicles at intracortical L4 synaptic boutons, $\sim 12,000$ for adult MFBs and $\sim 25,000$ for the calyx of Held. At individual AZs at intracortical L4 synaptic boutons, a nearly ninefold larger reserve pool was estimated when compared with the calyx of Held (see Fig. 12a).

In summary, although intracortical L4 synaptic boutons are relatively small, the size of their putative RRP, RP and reserve pools is comparable to or even larger than that of other CNS synapses. Hence, the high synaptic efficacy and strength at L4 synaptic boutons could be explained by the size of the three pools. Furthermore, the comparatively large reserve pool may prevent a complete depletion of the pool of synaptic vesicles by long-lasting repetitive stimulation due to a rapid recycling and refilling of the RRP and RP.

\section{Functional significance}

Taken together, three structural parameters underlie the unique functional behavior and high efficacy of intracortical L4 synaptic boutons in vitro: the large (perforated) release sites, the size of RRP, RP, and reserve pools per AZ and the tight ensheathment by fine astrocytic processes. Thus, multiple structural determinants converge on the generation of a 'conditional detonator' synapse perfectly adapted for the function of L4 as the first cortical information processing station in the cortical column.
Acknowledgments The excellent technical assistance by Eva Nicksch, Brigitte Marshallsay and Ulrike Bandelow is very much appreciated. We would like to thank Dr. Nicola Palomero-Gallagher for critically reading and helpful comments on a pre-final version of the manuscript. This work was supported by the IRTG 1328 (K.K.) and grants of the Deutsche Forschungsgemeinschaft DFG (J.L.). The authors declare no competing financial interests.

\section{References}

Abercrombie M (1946) Estimation of nuclear population from microtome sections. Anat Rec 94:239-246

Agmon A, Connors BW (1991) Thalamocortical responses of mouse somatosensory (barrel) cortex in vitro. Neuroscience 41:365-379

Ahmed B, Anderson JC, Douglas RJ, Martin KA, Nelson JC (1994) Polyneuronal innervation of spiny stellate neurons in cat visual cortex. J Comp Neurol 341:39-49

Ahmed B, Anderson JC, Martin KA, Nelson JC (1997) Map of the synapses onto layer 4 basket cells of the primary visual cortex of the cat. J Comp Neurol 380:230-242

Armstrong-James M, Fox K, Das-Gupta A (1992) Flow of excitation within rat barrel cortex on striking a single vibrissa. J Neurophysiol 68:1345-1358

Barth AL (2002) Differential plasticity in neocortical networks. Physiol Behav 77:545-550

Beierlein M, Gibson JR, Connors BW (2003) Two dynamically distinct inhibitory networks in layer 4 of the neocortex. J Neurophysiol 90:2987-3000

Benshalom G, White E (1986) Quantification of thalamocortical synapses with spiny stellate neurons in layer IV of mouse somatosensory cortex. J Comp Neurol 253:303-314

Brückner G, Brauer K, Hartig W, Wolff JR, Rickmann MJ, Derouiche A, Delpech B, Girard N, Oertel WH, Reichenbach A (1993) Perineuronal nets provide a polyanionic, glia-associated form of microenvironment around certain neurons in many parts of the rat brain. Glia 8:183-200

Bruno RM, Sakmann B (2006) Cortex is driven by weak but synchronously active thalamocortical synapses. Science 312:1622-1627

Constantinople CM, Bruno RM (2013) Deep cortical layers are activated directly by thalamus. Science 340:1591-1594

Cragg BG (1972) The development of synapses in cat visual cortex. Invest Ophthalmol 11:377-385

Cragg BG (1975) The development of synapses in the visual system of the cat. J Comp Neurol 160:147-166

Danbolt NC (2001) Glutamate uptake. Prog Neurobiol 65:1-105

Deller T, Korte M, Chabanis S, Drakew A, Schwegler H, Stefani GG, Zuniga A, Schwarz K, Bonhoeffer T, Zeller R, Frotscher M, Mundel P (2003) Synaptopodin-deficient mice lack a spine apparatus and show deficits in synaptic plasticity. Proc Natl Acad Sci USA 100:10494-10499

Egger V, Feldmeyer D, Sakmann B (1999) Coincidence detection and changes of synaptic efficacy in spiny stellate neurons in rat barrel cortex. Nat Neurosci 2:1098-1105

Feldmeyer D, Egger V, Lübke J, Sakmann B (1999) Reliable synaptic connections between pairs of excitatory layer 4 neurons within a single 'barrel' of developing rat somatosensory cortex. J Physiol 521:169-190

Feldmeyer D, Lübke J, Silver RA, Sakmann B (2002) Synaptic connections between layer 4 spiny neuron-layer $2 / 3$ pyramidal cell pairs in juvenile rat barrel cortex: physiology and anatomy of interlaminar signalling within a cortical column. J Physiol 538:803-822 
Feldmeyer D, Roth A, Sakmann B (2005) Monosynaptic connections between pairs of spiny stellate cells in layer 4 and pyramidal cells in layer $5 \mathrm{~A}$ indicate that lemniscal and paralemniscal afferent pathways converge in the infragranular somatosensory cortex. J Neurosci 25:3423-3431

Feldmeyer D, Brecht M, Helmchen F, Petersen CC, Poulet JF, Staiger JF, Luhmann HJ, Schwarz C (2013) Barrel cortex function. Prog Neurobiol 103:3-27

Freche D, Pannasch U, Rouach N, Holcman D (2011) Synapse geometry and receptor dynamics modulate synaptic strength. PLoS One 6:e25122

Geinisman Y (1993) Perforated axospinous synapses with multiple, completely partitioned transmission zones: probable structural intermediates in synaptic plasticity. Hippocampus 3:417-433

Geinisman Y, de Toledo-Morrell L, Morrell F (1991) Induction of long-term potentiation is associated with an increase in the number of axospinous synapses with segmented postsynaptic densities. Brain Res 566:77-88

Gray EG (1959a) Axo-somatic and axo-dendritic synapses of the cerebral cortex: an electron microscope study. J Anat 93:420-433

Gray EG (1959b) Electron microscopy of synaptic contacts on dendrite spines of the cerebral cortex. Nature 183:1592-1593

Hallermann S, Pawlu C, Jonas P, Heckmann M (2003) A large pool of releasable vesicles in a cortical glutamatergic synapse. Proc Natl Acad Sci USA 100:8975-8980

Harris RM, Woolsey TA (1983) Computer-assisted analyses of barrel neuron axons and their putative synaptic contacts. J Comp Neurol 220:63-79

Haydon PG, Carmignoto G (2006) Astrocyte control of synaptic transmission and neurovascular coupling. Physiol Rev 86:1009-1031

Helmstaedter M, Staiger JF, Sakmann B, Feldmeyer D (2008) Efficient recruitment of layer $2 / 3$ interneurons by layer 4 input in single columns of rat somatosensory cortex. J Neurosci 28:8273-8284

Helmstaedter M, Sakmann B, Feldmeyer D (2009) The relation between dendritic geometry, electrical excitability, and axonal projections of L2/3 interneurons in rat barrel cortex. Cereb Cortex 19:938-950

Holderith N, Lorincz A, Katona G, Rozsa B, Kulik A, Watanabe M, Nusser Z (2012) Release probability of hippocampal glutamatergic terminals scales with the size of the active zone. Nat Neurosci 15:988-997

Holtmaat AJ, Trachtenberg JT, Wilbrecht L, Shepherd GM, Zhang X, Knott GW, Svoboda K (2005) Transient and persistent dendritic spines in the neocortex in vivo. Neuron 45:279-291

Houades V, Koulakoff A, Ezan P, Seif I, Giaume C (2008) Gap junction-mediated astrocytic networks in the mouse barrel cortex. J Neurosci 28:5207-5217

Jacobs SE, Juliano SL (1995) The impact of basal forebrain lesions on the ability of rats to perform a sensory discrimination task involving barrel cortex. J Neurosci 15:1099-1109

Kätzel D, Zemelman BV, Buetfering C, Wolfel M, Miesenbock G (2011) The columnar and laminar organization of inhibitory connections to neocortical excitatory cells. Nat Neurosci 14:100-107

Koelbl C, Helmstaedter M, Lübke J, Feldmeyer D (2013) A barrelrelated interneuron in layer 4 of rat somatosensory cortex with a high intrabarrel connectivity. Cereb Cortex. doi:10.1093/cercor/ bht 263

Konur S, Yuste R (2004) Imaging the motility of dendritic protrusions and axon terminals: roles in axon sampling and synaptic competition. Mol Cell Neurosci 27:427-440

Kumar P, Ohana O (2008) Inter- and intralaminar subcircuits of excitatory and inhibitory neurons in layer $6 \mathrm{a}$ of the rat barrel cortex. J Neurophysiol 100:1909-1922
Land PW, Kandler K (2002) Somatotopic organization of rat thalamocortical slices. J Neurosci Methods 119:15-21

Le Meur K, Mendizabal-Zubiaga J, Grandes P, Audinat E (2012) GABA release by hippocampal astrocytes. Front Comput Neurosci 6:1-10

Lee CC, Sherman SM (2008) Synaptic properties of thalamic and intracortical inputs to layer 4 of the first- and higher-order cortical areas in the auditory and somatosensory systems. J Neurophysiol 100:317-326

Lübke J, Feldmeyer D (2007) Excitatory signal flow and connectivity in a cortical column: focus on barrel cortex. Brain Struct Funct 212:3-17

Lübke J, Egger V, Sakmann B, Feldmeyer D (2000) Columnar organization of dendrites and axons of single and synaptically coupled excitatory spiny neurons in layer 4 of the rat barrel cortex. J Neurosci 20:5300-5311

Lübke J, Roth A, Feldmeyer D, Sakmann B (2003) Morphometric analysis of the columnar innervation domain of neurons connecting layer 4 and layer $2 / 3$ of juvenile rat barrel cortex. Cereb Cortex 13:1051-1063

Matz J, Gilyan A, Kolar A, McCarvill T, Krueger SR (2010) Rapid structural alterations of the active zone lead to sustained changes in neurotransmitter release. Proc Natl Acad Sci USA 107:8836-8841

Meyer HS, Wimmer VC, Hemberger M, Bruno RM, de Kock CP, Frick A, Sakmann B, Helmstaedter M (2010) Cell type-specific thalamic innervation in a column of rat vibrissal cortex. Cereb Cortex 20:2287-2303

Meyer HS, Schwarz D, Wimmer VC, Schmitt AC, Kerr JN, Sakmann B, Helmstaedter M (2011) Inhibitory interneurons in a cortical column form hot zones of inhibition in layers 2 and 5A. Proc Natl Acad Sci USA 108:16807-16812

Min R, Nevian T (2012) Astrocyte signaling controls spike timingdependent depression at neocortical synapses. Nat Neurosci 15:746-753

Mironov SL (2006) Spontaneous and evoked neuronal activities regulate movements of single neuronal mitochondria. Synapse 59:403-411

Mironov SL, Symonchuk N (2006) ER vesicles and mitochondria move and communicate at synapses. J Cell Sci 119:4926-4934

Mukherjee K, Yang X, Gerber SH, Kwon HB, Ho A, Castillo PE, Liu X, Südhof TC (2010) Piccolo and bassoon maintain synaptic vesicle clustering without directly participating in vesicle exocytosis. Proc Natl Acad Sci USA 107:6504-6509

Müller J, Reyes-Haro D, Pivneva T, Nolte C, Schaette R, Lübke J, Kettenmann H (2009) The principal neurons of the medial nucleus of the trapezoid body and $\mathrm{NG}_{2}^{(+)}$glial cells receive coordinated excitatory synaptic input. J Gen Physiol 134:115-127

Nicol MJ, Walmsley B (2002) Ultrastructural basis of synaptic transmission between endbulbs of Held and bushy cells in the rat cochlear nucleus. J Physiol 539:713-723

Nicoletta N, Fenghua C, Gregers W, Maurizio P, Randel NJ (2013) A new efficient method for synaptic vesicle quantification reveals differences between medial prefrontal cortex perforated and nonperforated synapses. J Comp Neurol. doi:10.1002/cne.23482

Oliet SH, Piet R, Poulain DA, Theodosis DT (2004) Glial modulation of synaptic transmission: insights from the supraoptic nucleus of the hypothalamus. Glia 47:258-267

Pannasch U, Freche D, Dallérac G, Ghézali G, Escartin C, Ezan P, Cohen-Salmon M, Benchenane K, Abudara V, Dufour A, Lübke JHR, Déglon N, Knott G, Holcman D, Rouach N (2014) Connexin 30 sets synaptic strength by controlling astroglial synapse invasion. Nat Neurosci 17:549-558

Peters A, Kaiserman-Abramof IR (1969) The small pyramidal neuron of the rat cerebral cortex. The synapses upon dendritic spines. Z Zellforsch Mikrosk Anat 100:487-506 
Pozzan T, Magalhaes P, Rizzuto R (2000) The comeback of mitochondria to calcium signalling. Cell Calcium 28:279-283

Radnikow G, Günter RH, Günter RH, Marx M, Feldmeyer D (2012) Morphofunctional mapping of cortical networks in brain slice preparations using paired electrophysiological recordings. In: Fellin T, Halassa M (eds) Neuromethods: neuronal network analysis. Humana Press, Springer Science and Business Media, LLC 2011, New York, pp 405-431

Ramirez A, Pnevmatikakis EA, Merel J, Paninski L, Miller KD, Bruno RM (2014) Spatiotemporal receptive fields of barrel cortex revealed by reverse correlation of synaptic input. Nat Neurosci 17:866-875

Reynolds ES (1963) The use of lead citrate at high $\mathrm{pH}$ as an electronopaque stain in electron microscopy. J Cell Biol 17:208-212

Rizzoli SO, Betz WJ (2004) The structural organization of the readily releasable pool of synaptic vesicles. Science 303:2037-2039

Rizzoli SO, Betz WJ (2005) Synaptic vesicle pools. Nat Rev Neurosci 6:57-69

Rizzuto R, Bernardi P, Pozzan T (2000) Mitochondria as all-round players of the calcium game. J Physiol 529:37-47

Rodríguez-Contreras A, van Hoeve JS, Habets RL, Locher H, Borst JG (2008) Dynamic development of the calyx of Held synapse. Proc Natl Acad Sci USA 105:5603-5608

Rollenhagen A, Lübke JHR (2006) The morphology of excitatory central synapses: from structure to function. Cell Tissue Res 326:221-237

Rollenhagen A, Sätzler K, Rodriguez EP, Jonas P, Frotscher M, Lübke JHR (2007) Structural determinants of transmission at large hippocampal mossy fiber synapses. J Neurosci 27:10434-10444

Rollenhagen A, Klook K, Suchmann C, Kasugai Y, Ferraguti F, Shigemoto R, Lübke JHR (2012) Differential expression and distribution patterns of AMPA and NMDA receptors and their subunits at layer 4 and layer 5 synapses in the rat somatosensory cortex. Soc Neurosci Abstr, 43.12 (online)

Rosenmund C, Stevens CF (1996) Definition of the readily releasable pool of vesicles at hippocampal synapses. Neuron 16:1197-1207

Rowland KC, Irby NK, Spirou GA (2000) Specialized synapseassociated structures within the calyx of Held. J Neurosci 20:9135-9144

Sahara Y, Takahashi T (2001) Quantal components of the excitatory postsynaptic currents at a rat central auditory synapse. J Physiol 536:189-197

Sätzler K, Söhl LF, Bollmann JH, Borst JG, Frotscher M, Sakmann B, Lübke JHR (2002) Three-dimensional reconstruction of a calyx of Held and its postsynaptic principal neuron in the medial nucleus of the trapezoid body. J Neurosci 22:10567-10579

Saviane C, Silver RA (2006) Fast vesicle reloading and a large pool sustain high bandwidth transmission at a central synapse. Nature 439:983-987

Schikorski T, Stevens CF (2001) Morphological correlates of functionally defined synaptic vesicle populations. Nat Neurosci 4:391-395

Schneggenburger R, Sakaba T, Neher E (2002) Vesicle pools and short-term synaptic depression: lessons from a large synapse. Trends Neurosci 25:206-212

Schoch S, Gundelfinger ED (2006) Molecular organization of the presynaptic active zone. Cell Tissue Res 326:379-391

Schoonover CE, Tapia JC, Schilling VC, Wimmer V, Blazeski R, Zhang W, Mason CA, Bruno RM (2014) Comparative strength and dendritic organization of thalamocortical and corticocortical synapses onto excitatory layer 4 neurons. J Neurosci 34:6746-6758

Shapira M, Zhai RG, Dresbach T, Bresler T, Torres VI, Gundelfinger ED, Ziv NE, Garner CC (2003) Unitary assembly of presynaptic active zones from Piccolo-Bassoon transport vesicles. Neuron $38: 237-252$
Sherman SM, Guillery RW (1996) Functional organization of thalamocortical relays. J Neurophysiol 76:1367-1395

Silver RA, Lübke J, Sakmann B, Feldmeyer D (2003) Highprobability uniquantal transmission at excitatory synapses in barrel cortex. Science 302:1981-1984

Simons DJ, Woolsey TA (1984) Morphology of Golgi-Cox-impregnated barrel neurons in rat SmI cortex. J Comp Neurol 230:119-132

Somogyi P, Hodgson AJ (1985) Antisera to gamma-aminobutyric acid. III. Demonstration of GABA in Golgi-impregnated neurons and in conventional electron microscopic sections of cat striate cortex. J Histochem Cytochem 33:249-257

Staiger JF, Flagmeyer I, Schubert D, Zilles K, Kötter R, Luhmann HJ (2004) Functional diversity of layer IV spiny neurons in rat somatosensory cortex: quantitative morphology of electrophysiologically characterized and biocytin labeled cells. Cereb Cortex 14:690-701

Staiger JF, Zuschratter W, Luhmann HJ, Schubert D (2009) Local circuits targeting parvalbumin-containing interneurons in layer IV of rat barrel cortex. Brain Struct Funct 214:1-13

Südhof TC (2012) The presynaptic active zone. Neuron 75:11-25

Umeda T, Ebihara T, Okabe S (2005) Simultaneous observation of stably associated presynaptic varicosities and postsynaptic spines: morphological alterations of CA3-CA1 synapses in hippocampal slice cultures. Mol Cell Neurosci 28:264-274

Verstreken P, Ly CV, Venken KJ, Koh TW, Zhou Y, Bellen HJ (2005) Synaptic mitochondria are critical for mobilization of reserve pool vesicles at Drosophila neuromuscular junctions. Neuron 47:365-378

von Gersdorff H, Borst JG (2002) Short-term plasticity at the calyx of held. Nat Rev Neurosci 3:53-64

Wanaverbecq N, Bodor AL, Bokor H, Slezia A, Luthi A, Acsady L (2008) Contrasting the functional properties of GABAergic axon terminals with single and multiple synapses in the thalamus. J Neurosci 28:11848-11861

Watanabe S, Rost BR, Camacho-Perez M, Davis MW, SohlKielczynski B, Rosenmund C, Jorgensen EM (2013) Ultrafast endocytosis at mouse hippocampal synapses. Nature 504:242-247

White EL, Benshalom G, Hersch SM (1984) Thalamocortical and other synapses involving nonspiny multipolar cells of mouse SmI cortex. J Comp Neurol 229:311-320

Xu-Friedman MA, Regehr WG (2003) Ultrastructural contributions to desensitization at cerebellar mossy fiber to granule cell synapses. J Neurosci 23:2182-2192

Xu-Friedman MA, Regehr WG (2004) Structural contributions to short-term synaptic plasticity. Physiol Rev 84:69-85

Xu-Friedman MA, Harris KM, Regehr WG (2001) Three-dimensional comparison of ultrastructural characteristics at depressing and facilitating synapses onto cerebellar Purkinje cells. J Neurosci 21:6666-6672

Yu C, Derdikman D, Haidarliu S, Ahissar E (2006) Parallel thalamic pathways for whisking and touch signals in the rat. PLoS Biol 4:e124

Zhang ZW, Deschenes M (1997) Intracortical axonal projections of lamina VI cells of the primary somatosensory cortex in the rat: a single-cell labeling study. J Neurosci 17:6365-6379

Zhao S, Studer D, Graber W, Nestel S, Frotscher M (2012a) Fine structure of hippocampal mossy fiber synapses following rapid high-pressure freezing. Epilepsia 53(Suppl. 1):4-8

Zhao S, Studer D, Chai X, Graber W, Brose N, Nestel S, Young C, Rodriguez EP, Sätzler K, Frotscher M (2012b) Structural plasticity of hippocampal mossy fiber synapses as revealed by high-pressure freezing. J Comp Neurol 520:2340-2351 